\title{
A Distributed Polarizing Transmission System for Frequency Se- lective Fading Channels
}

\author{
${ }^{1}$ Tinuola Olayinka Coker, ${ }^{2}$ Joulani Shadi Muhammad Jamal, ${ }^{3}$ Biniyame Mulatu \\ Yilma and ${ }^{4}$ Redon Dimroci
}

\begin{abstract}
Motivated by Arikan's channel polarization that shows the occurrence of capacity-achieving code sequences, we address the scheme design issues by switching to polarizing frequency selective fading channels while transmitting information symbols in a source-relay-destination MIMO-OFDM relay communication system. A simple polar-and-forward (PF) MIMO relay scheme, with source node polar coding and relay nodes polar coding, is proposed to provide an alternative solution for transmitting with higher reliability than the conventional decode-and-forward/amplify-and-forward (DF/AF) relay schemes. In the proposed scheme, OFDM modulator is implemented at source node, some simple operations, namely time reversion, complex conjugation and polarization, are implemented at relay nodes, and the cyclic prefix $(C P)$ removal is performed at destination node. It is divided into two symmetrical polarizing relay systems, i.e., the down-polarizing system and the up-polarizing system, which result in different capacities for the polar system. We analyze the bit error rate $(B E R)$ performance with the fixed polar system equipped with four OFDM blocks, which is an idea approach to select signal sequences that tend to polarize in terms of the reliability under certain combining and splitting the transmitted OFDMs in the frequency selective fading (FSF) channels. The polar system has a salient recursiveness feature, and thus the transmitted information signals embedded in the polar code can be decoded with a low-complexity decoder.
\end{abstract}

\section{Introduction}

The channel polarization shows an attractive construction of provably capacity-achieving coding sequences [1]-[6]. It has provided an attempt method to meet this elusive goal for multi- fold binary-input discrete memoryless channels, where channel combining and splitting operations were applied to improve its symmetric capacity [1], [2]. Actually, the polarization of multiple channels is a commonplace phenomenon and thus it is almost impossible to avoid as long as several channels are synthesized in a proper density with certain arrangements. During the past decade, the multiple-input multi-output (MIMO) communication system has been well studied to promise significants of the increasing spectral effciency, channel capacity and link reliability [7][11]. It shows that the coding gain and diversity can be simultaneously achieved with suitable coding schemes. As the MIMO techniques grown up, researchers have been exploring new communication paradigms. A potential proposal is the so-called wireless relay system, which provides the reliable transmission, high throughput and broad coverage for wireless network [12], [19], and [20]. The eminent merits of a MIMO wireless system lie in its potential temporal diversity gain, spatial diversity gain and multiplexing gain to enhance link reliability. This elegant technique can be further exploited to explore the potential spatial and temporal diversity on flat-fading or frequency selective fading (FSF) channels with some proper transmission schemes, such as space-time (ST) coding [13], space-frequency (SF) coding and space-time-frequency (STF) coding [14]-[18]. It is shown that the coding gain and diversity can be simultaneously achieved with suitable coding schemes. Unfortunately, as the number of transmit antennas becomes large, the complexity of decoding increases, which makes the design of coding or modulating schemes difficult.

MIMO relay communications, together with the orthogonal frequency division multiplexing (OFDM) techniques, present an effective way of increasing reliability as well as achievable rates in next generation wireless networks. Cooperative diversity is usually achieved through relay nodes that help the source node forwarding its information. Deploying proper relays between source node and destination node can not only overcome shadowing due to inevitable obstacles, but also reduce the transmit power from the source node. In the MIMO- OFDM relay system, two or more nodes share and transmit jointly their information symbols in a multi-antenna array, which enables the high data rate and diversity gain. A usual approach to share information is to tune in the transmitted signals and process the whole (or partial) received information in regenerative or non-regenerative way. The former employs a decode-and-forward (DF) relay scheme in which each relay decodes the original information from the source and forwards it to the destination [12]. Unfortunately, since channels are usually noisy and fading, the processed information signals are not perfect. Therefore, we have to study a possible coding or modulating strategy to improve its performance that makes a merit of relay system. In the latter scheme it exploits an amplify-and-forward (AF) scheme to amplify and retransmit the 
scaled signals without any attempt to decode the original information [21], [22]. In the light of superiority of these relay strategies with the availability of CSI, we consider the coding design of the MIMO-OFDM relay system for the FSF channels with the fixed gain relaying scheme using the polar- and-forward (PF) relay technique, in which each relay node encodes and retransmits the partial signals with the fixed power constraint. A key feature of this scheme is that we do not require relays to decode. Only a simple processing operation is done at each relay, which makes the transmission much simple and hence can avoid imposing bottlenecks on the data rate.

Recently, significant efforts have been related to the increasing capacity [23] or the optimal design of the relay system [24] in terms of the DF/AF relay schemes based on a scenario equipped with single or multiple antennas. However, further improvement should be sought in these relay systems, in which the loss of the signal rate is boosted as the number of relay nodes along with antennas increases. While a key component relay design is to optimize the precoding of source and relay in benefits of multiple antennas and multiple OFDM symbols, how to design the MIMO-OFDM relay system to achieve high reliability with low-decoding complexity via a coding approach becomes a challenge.

The problem with the previous relay system is the data rate loss as the number of relay nodes increases. This leads to the use of polar coding sequences in MIMO-OFDM system, where relay nodes are allowed to simultaneously transmit the same OFDM systems over the FSF channels. We consider a simple design of the relay system that achieves the fascinating symmetric capacity of the FSF channels based on polar coding with a successive interference cancellation (SIC) decoder at destination node, which is motivated by the fascinating Shanon's channel coding theorem [25]. It is an extension of work where OFDM combining and splitting are used for recursive code construction with the SIC decoding, which are essential characters of the polar coding sequences [1]-[6]. This is an idea approach to construct code sequences as combining and splitting OFDM for the FSF channels to increase its reliability.

Furthermore, we establish an analytical framework that illustrates the potential bit error rate (BER) performance to be achieved from the polar MIMO-OFDM relay system. We argue that the present system may increase the symmetric capacity under a low computation complexity of the SIC decoding due to the fact that a large number OFDM symbols may be equipped for the polarizing FSF channels that tend to polarize under certain OFDM combining and splitting operations.

This paper is organized as follows. In Section II, we describe the polar MIMO relay system with two switching communication model, the down-polarizing system and the up-polarizing system. In Section III, we systematically study the design of an simple PF scheme with space-time-frequency (STF) transmission for down-polarizing and up-polarizing FSF channels. Some simulation results are also depicted in order to show the BER performance behavior and robustness of this polar MIMO-OFDM relay system. Finally, conclusions are drawn in Section IV.

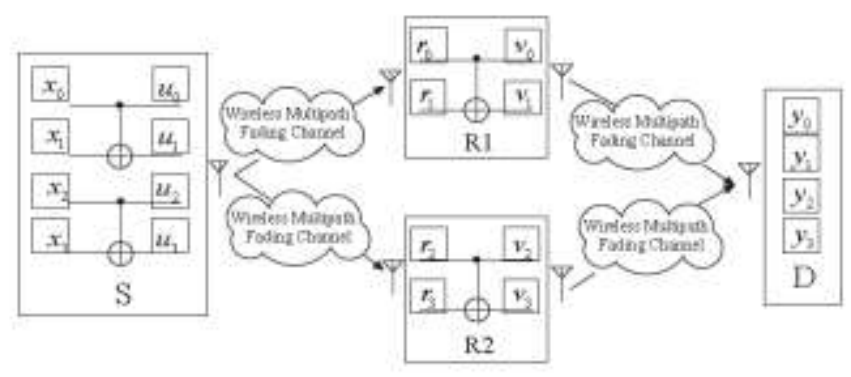

a)

Down-polarizing OFDM Blocks for Distributed System

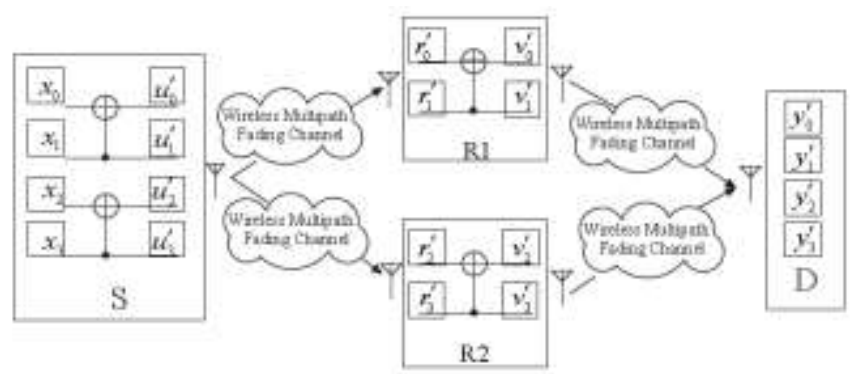

b) Up-polarizing OFDM blocks for Distributed System

Fig. 1. The relay communication system based on the polarizing MIMOOFDM channels with two models: (a) denotes the down-polarizing communication model; and (b) denotes the up-polarizing communication model. 
Some notations are defined throughout this paper as follows:

- $\quad \mathbb{C}$ : complex number field;

- $\mathbb{Z}_{\mathrm{T}}$ : finite non-negative integer set $\{0,1, \cdots, \mathrm{T}-1\}$;

- Bold faced uppercase letters, such as A: matrices;

- tr: trace of a matrix;

- Bold faced down-case letters, such as a: column vectors;

- Superscripts $(\cdot)^{\mathrm{T}},(\cdot)^{\mathrm{H}}$, and $(\cdot)^{*}$ : transpose, complex, conjugate transpose, complex conjugate, respectively;

- $\|\cdot\|_{\mathrm{F}}$ : Frobenius norm of a matrix;

- $\mathrm{E}[\square]$ ]: expectation of variable $\square$;

- $\otimes$ : the Kronecker product;

- $\quad$ : the Hadamard product, i.e., the component-wise product;

- $\mathbf{I}_{\mathrm{n}}$ : identity matrix of size $\mathrm{n} \times \mathrm{n}$;

- $\operatorname{diag}\left(\mathbf{d}_{0}, \ldots, \mathbf{d}_{\mathrm{N}-1}\right)$ : a diagonal matrix with diagonal entries $\mathbf{d}_{0}, \cdots, \mathbf{d}_{\mathrm{N}-1}$.

\section{Channel Polarization: Down-Polarizing And Up-Polarizing Mimo-Ofdm Relay System}

We consider the distributed wireless system based on OFDM modulation with $\mathrm{N}$ subcarriers. There is one source node $S$, one destination node $D$, and two relay nodes $R \triangleq,\{R 1, R 2\}$, as shown in Fig.1. There is only one antenna at all nodes $\mathrm{S}, \mathrm{R}$ and $\mathrm{D}$, respectively. This assumption is applicable for any nodes equipped with multiple antennas. We consider a scenario where $\mathrm{N}_{\mathrm{s}}$ OFDM symbols are transmitted for $\mathrm{N}_{\mathrm{s}}=2^{\mathrm{n}}$. The design of the relay scheme that can mitigate relay synchronization errors is considered. Each relay node $R_{k}, \forall k \in\{1,2\}$, is assumed to be capable of processing the OFDM symbols independently and correctly. The average transmit power at source node $S$ is $p_{t}$. The relay scheme is half-duplex, meaning that $S$ and $R$ do not transmit and receive simultaneously. The $\mathrm{N}_{\mathrm{s}}$ independent OFDM symbols are transmitted simultaneously from source node $\mathrm{S}$ to destination node $\mathrm{D}$ in two stages. In the first stage the initial signal OFDM symbols are polarized and transmitted from source node $S$ to each relay node $R_{k}, \forall k \in\{1,2\}$. In the second stage each relay node $R_{k}$ forwards the (partial) signal vector received from source node $S$ to destination node $D$ while source node $S$ keeps silent. We further assume that each single-link between a pair of transmit antenna and receive antenna is frequency selective Rayleigh fading with $\mathrm{L}$ independent propagation, which experiences quasi-static and remains unchanged in certain blocks. Denote the fading coefficient from source node $S$ to relay node $R_{k}$ as $h_{S_{k}}=\varphi_{k}$ and the fading coefficient from relay node $R_{k}$ to destination node $D$ as $h_{R_{k}} D=\kappa_{k}$. Assume that $\varphi_{k}$ and $\kappa_{k}, \forall k \in\{1,2\}$, are independent zero mean complex Gaussian random variables. Two channel impulse responses $\varphi_{k}(t)$ from source node $S$ to destination node $\mathrm{R}$ are written as:

$$
\phi_{k}(t)=\sum_{l=0}^{L-1} \alpha_{s k}(l) \delta\left(t-\tau_{l, s k}\right),
$$

where $\alpha_{\mathrm{sk}}(\square)$ represents the channel coefficient of the $\square^{\text {th }}$ path of the channels, and T $\square$,sk is the corresponding path delay. Each channel coefficient $\alpha_{\mathrm{sk}}(\square)$ is modelled as zero mean complex Gaussian random variables with variance $\sigma_{\square}^{2}$,sk such that $\Sigma_{l=0}^{L-1} \sigma_{l, s k}^{2}=1$. We also assume that $\alpha_{\mathrm{sk}}(\square)$ are i.i.d. random variables for any $(k, \square)$. Similarly, other two channel impulse responses $\kappa_{k}(t)$ from relay node $R_{k}$ to destination node $\mathrm{D}$ are written as:

$$
K_{k}(t)=\sum_{l=0}^{L-1} \alpha_{s k}(l) \delta\left(t-\tau_{l, s k}\right),
$$

where $\operatorname{ark}(\mathrm{l})$ represents the channel coefficient of the lth path of the channels, and $\mathrm{ll}$,rk is the corresponding path delay. Each channel coefficient ark(l) is also modelled as zero mean complex Gaussian random variables with variance $\sigma 2$ l,rk such that $\Sigma_{l=0}^{L-1} \sigma_{l, r k}^{2}=1$. In addition, we denote the average power for one transmission of each relay Rk as pr. The constraint on the total network power is $\mathrm{p}=\mathrm{pt}+2 \mathrm{pr}$. We also adopt the power allocation strategy suggested in [26], and thus have

$$
\mathrm{p}_{\mathrm{t}}=2 \mathrm{p}_{\mathrm{r}}=\mathrm{p} / 2 \text {. }
$$

The MIMO-OFDM channel model, denoted by $\mathbf{H} \in \mathbb{C}^{2 \times 2}$, is created between source node $\mathrm{S}$ and relay nodes $\mathrm{R}$, and $\mathbf{K} \in \mathbb{C}^{2 \times 2}$ between $\mathrm{R}$ and D. Here entries of $\mathbf{H}$ and $\mathbf{K}$ are assumed independent and identically distributed 
(i.i.d.) with distribution $\mathrm{CN}(0,1)$. For the distributed MIMO-OFDM relay wireless system with source-relaydestination triplet structure, it is equivalent to two partial MIMO-OFDM wireless systems. One part has the MIMO channel model $\mathrm{H}$, i.e.,

$\mathbf{H}=\operatorname{diag}\left(\varphi_{1}, \varphi_{2}\right)$,

and another part $\mathbf{K}$ is given by

$\mathbf{K}=\operatorname{diag}\left(\kappa_{1}, \kappa_{2}\right)$.

Based on the MIMO-ODFM relay channels $\mathbf{H}$ and $\mathbf{K}$ in (4) and (5), we design the polar system for the transmission of the signal vector $\mathbf{x}$, in which we switch to the polar system in four consecutive time slots, i.e., downpolarizing and up-polarizing communications in turn. The system has two transmission phases. In phase 1, the source node broadcasts four OFDM symbols that are first polarized at source node $S$ to each relay node $R_{k}$. In phase 2, source node $S$ stops the transmission and each relay node $R_{k}$ that polarizes the received symbols for the second time and retransmits the resulting symbols to destination node $\mathrm{D}$.

\section{A. Down-polarizing MIMO-OFDM Relay System}

At source node $\mathrm{S}$ the transmitted information is modulated into complex symbols $\mathrm{x}_{\mathrm{ij}}$ and then each $\mathrm{N}$ modulated symbol as a block are poured into an OFDM modulator of $\mathrm{N}$ subcarriers. Denote four consecutive OFDM blocks by $\mathrm{x}_{\mathrm{i}}=\left(\mathrm{x}_{\mathrm{i}, 0}, \mathrm{x}_{\mathrm{i}, 1}, \ldots, \mathrm{x}_{\mathrm{i}}, \mathrm{N}-1\right)^{\mathrm{T}}, \forall \mathrm{i} \in \mathbb{Z}_{4}$. We define $\mathrm{x}_{\mathrm{i}}+\mathrm{x}_{\mathrm{j}}=\left(\mathrm{x}_{\mathrm{i}, 0}+\mathrm{x}_{\mathrm{j}, 0}, \mathrm{x}_{\mathrm{i}, 1}+\mathrm{x}_{\mathrm{j}, 1}, \ldots, \mathrm{x}_{\mathrm{i}}, \mathrm{N}-1+\right.$ $\left.\mathrm{x}_{\mathrm{j}}, \mathrm{N}-1\right) \mathrm{T}, \forall \mathrm{i}, \mathrm{j} \in \mathbb{Z}_{4}$, for polarization calculation.

In the first time slot, four consecutive OFDM blocks are processed with the down-polarizing $4 \times 4$ matrix $\mathbf{Q}_{4}$ at source node $\mathrm{S}$, i.e.,

$\mathbf{U}=\mathbf{X} \mathbf{Q}_{4}$,

where $\mathbf{U}=\left(\mathrm{u}_{0}, \mathrm{u}_{1}, \mathrm{u}_{2}, \mathrm{u}_{3}\right)$ denotes the polarized matrix of size $\mathrm{N} \times 4, \mathbf{X}=\left(\mathrm{x}_{0}, \mathrm{x}_{1}, \mathrm{x}_{2}, \mathrm{x}_{3}\right)$ denotes the signal matrix of size $\mathrm{N} \times 4$ corresponding to four OFDM blocks, the polar matrix $\mathbf{Q}_{\mathbf{4}}$ is given by $\mathbf{Q}_{\mathbf{4}}=\mathbf{I}_{\mathbf{2}} \otimes \mathbf{Q}_{2}$. Here matrix $\mathbf{Q}_{\mathbf{2}}$ is a down-polarizing matrix defined as Arikan's fashion [1], i.e.,

$\mathbf{Q}_{2}=$

Therefore, we have $\mathrm{u}_{2 \mathrm{k}-2}=\chi_{2 \mathrm{k}-2}$ and $\mathrm{u}_{2 \mathrm{k}-1}=\chi_{2 \mathrm{k}-2}+\chi_{2 \mathrm{k}-1}$, for $\forall \mathrm{k} \in\{1,2\}$.

$$
\left(\begin{array}{ll}
1 & 1 \\
0 & 1
\end{array}\right)
$$

In the OFDM modulator, the four consecutive blocks are modulated by N-point FFT. Then each block is precoded by a cyclic prefix (CP) with length $1_{\text {cp }}$. Thus each OFDM symbol consists of $\mathrm{Ls}=\mathrm{N}+\mathrm{l}_{\mathrm{cp}}$ samples. Finally, four OFDM symbols are broadcasted to two relay nodes. Denote by $\tau_{\mathrm{sd} 2}$ the overall relative delay from source node $S$ to relay node $R_{2}$, and then to destination node $\mathrm{D}$, where the relative delay means it is relative to relay node $R_{1}$. In order to combat against both frequency selective fading channels and timing errors, we assume that $1_{\mathrm{cp}} \geq \max _{\mathrm{l}, \mathrm{k}}\left\{\tau l_{\mathrm{sk}}+\tau l_{\mathrm{rk}}+\tau_{\mathrm{sd} 2}\right\}$. Denote four consecutive OFDM symbols by $\breve{\mathrm{u}}_{\mathrm{i}}, \forall \mathrm{i} \in \mathbb{Z}_{4}$, where $\breve{\mathrm{u}}_{\mathrm{i}}$ consists of $\mathrm{FFT}\left(\mathrm{u}_{\mathrm{i}}\right)$ and the corresponding CP.

At each relay $R_{k}$, the received noisy signals will be simply processed and forwarded to destination node D. Assume the channel coefficients are constant during four OFDM symbol intervals.

We define two processed vectors $\breve{\mathrm{u}}_{1}=\quad$ and $\breve{\mathrm{u}} 2=$

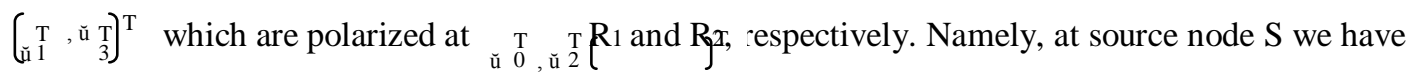

$$
\begin{array}{ll}
\mathrm{u}_{1}=\chi^{0^{\mathrm{T}}, \chi_{3}^{\mathrm{T}}} & \left.\left(\left(\chi_{0}+\chi_{1}\right)^{\mathrm{T}} \quad \chi_{2}+\chi_{3}\right)^{\mathrm{T}}\right)^{\mathrm{T}},
\end{array}
$$

and consequently

$\breve{\mathrm{u}}_{1}=\left(\operatorname{FFT}\left(\chi_{0}\right)^{\mathrm{T}}, \operatorname{FFT}\left(\chi_{2}\right)^{\mathrm{T}}\right)^{\mathrm{T}}$,

$\breve{\mathrm{u}}_{2}=\left(\operatorname{FFT}\left(\chi_{0}+\chi_{1}\right)^{\mathrm{T}}, \operatorname{FFT}\left(\chi_{2}+\chi_{3}\right)^{\mathrm{T}}\right)^{\mathrm{T}}$.

Therefore, the received signals at $\mathrm{R}_{\mathrm{k}}, \forall \mathrm{k} \in\{1,2\}$, for four successive OFDM symbol durations can be given by $\breve{\mathrm{r}}_{\mathrm{kO}}=\sqrt{\mathrm{pt}} \breve{\mathrm{u}}_{0} \circledast \square_{\mathrm{k}}+\stackrel{\underline{\mathrm{v}}}{\mathrm{n} \mathrm{k} 0}$ 


$$
\begin{aligned}
& \check{\mathrm{r}}_{\mathrm{ko1}}=\sqrt{ } \mathrm{pt} \breve{\mathrm{u}}_{1} \circledast \square_{\mathrm{k}}+\frac{\mathrm{v}}{\mathrm{n} k 1} \\
& \check{\mathrm{r}}_{\mathrm{k} 2}=\sqrt{\mathrm{pt}} \breve{\mathrm{u}}_{2} \circledast \square_{\mathrm{k}}+\frac{\underline{\mathrm{v}} \mathrm{k} 2}{\check{\mathrm{r}}_{\mathrm{k} 3}}=\sqrt{\mathrm{p} t} \breve{\mathrm{u}}_{1} \circledast \square_{\mathrm{k}}+\frac{\mathrm{v}_{\mathrm{n}} \mathrm{k} 3}{}
\end{aligned}
$$

TABLE I

IMPLEMENTATION OF THE PF SCHEME FOR THE DOWN-POLARIZED SYSTEM AT RELAY NODES. OF ${ }_{i}$ DENOTE THE $i^{\text {th }}$ OFDM BLOCK.

\begin{tabular}{|c|c|c|c|c|}
\hline & Polar $\mathrm{R}_{1}$ & Polar $\mathrm{R}_{1}$ & Process $\mathrm{R}_{1}$ & Process $\mathrm{R}_{2}$ \\
\hline $\mathrm{OF}_{0}$ & $\check{\mathbf{r}}_{10}$ & $\check{\mathrm{r}}_{20}$ & $\zeta\left(\check{\mathrm{r}}_{10}\right)$ & 0 \\
\hline $\mathrm{OF}_{1}$ & $\check{\mathrm{r}}_{11}$ & $\check{\mathrm{r}}_{21}$ & 0 & $\check{\mathrm{r}}_{21}$ \\
\hline $\mathrm{OF}_{2}$ & $\check{\mathrm{r}}_{10}+\check{\mathrm{r}}_{12}$ & $\check{\mathrm{r}}_{22}$ & $\zeta\left(\check{\mathrm{r}}_{10}+\check{\mathrm{r}}_{12}\right)$ & 0 \\
\hline $\mathrm{OF}_{3}$ & $\check{\mathrm{r}}_{13}$ & $\check{\mathrm{r}}_{23}+\check{\mathrm{r}}_{21}$ & 0 & $\left(\check{\mathrm{r}}_{23}+\check{\mathrm{r}}_{21}\right)^{*}$ \\
\hline
\end{tabular}

where $\sqrt{ } \mathrm{pt}$ is the transmission power at source node $\mathrm{S}, \varphi_{\mathrm{k}}$ is an $\mathrm{L} \times 1$ vector defined as $\varphi_{\mathrm{k}}=\left(\alpha_{\mathrm{sk}}(0), \alpha_{\mathrm{sk}}(1), \ldots\right.$, $\left.\alpha_{\text {sk }}(L-1)\right), \circledast$ denotes the linear convolution, and $\underset{\mathrm{n} k}{\mathrm{v}}, \forall \mathrm{i} \in \mathbb{Z}_{4}$, denotes the corresponding additive white Gaussian noise (AWGN) at relay node $\mathrm{R}_{\mathrm{k}}$ with zero-mean and unit-variance, in four successive OFDM symbol durations.

Then each relay node $R_{k}$ polarizes, processes and forwards the received noisy signals as shown in Table $I$, where $\zeta(\cdot)$ denotes the time-reversal of the signals, i.e., $\zeta\left(\breve{\mathrm{r}}_{\mathrm{ki}}(\square)\right) \triangleq \breve{\mathrm{r}}_{\mathrm{ki}}\left(\mathrm{L}_{\mathrm{s}}-\square\right), \forall \square \in \mathbb{Z}_{\mathrm{Ls}}$, and hence $\zeta\left(\breve{\mathrm{r}}_{\mathrm{ki}}\left(\mathrm{L}_{\mathrm{s}}\right)\right)$ $=\check{\mathrm{r}}_{\mathrm{ki}}(0), \forall \mathrm{k} \in\{1,2\}$ and $\forall \mathrm{i} \in \mathbb{Z} 4$. Denote by $\square_{0} \triangleq \zeta\left(\check{\mathrm{r}}_{10}\right), \square_{1} \triangleq \zeta\left(\check{\mathrm{r}}_{10}+\check{\mathrm{r}}_{12}\right), \square_{2} \triangleq \check{\mathrm{r}}_{21}$ and $\square_{3} \triangleq\left(\check{\mathrm{r}}_{21}+\check{\mathrm{r}}_{23}\right) *$. For the $\square$ th subcarrier of $\square_{\mathrm{i}}$ we also take the notations $\square_{\mathrm{i}}, \square \triangleq \square_{\mathrm{i}}(\square), \forall \in \mathbb{Z}_{\mathrm{N} . \Delta}$

After performing the above-mentioned processing operations, each relay node $R_{k}$ amplifies the yielded symbols with a scalar $\lambda=\sqrt{ } \mathrm{pr} /\left(\mathrm{p}_{\mathrm{t}}+1\right)$ while remaining the average transmission power $\mathrm{p}_{\mathrm{r}}$. In order to make the PF scheme available for the FSF channels, it is required that for each relay $R_{k}$ it can only implement the time reversal operation $\zeta(\cdot)$ or the complex conjugation operation $(\cdot)^{*}$ on the received OFDM symbols.

At destination node $\mathrm{D}$, the $\mathrm{CP}$ is removed for each OFDM symbol. We note that relay node R1 implements the time reversions of the noisy signals including both information symbols and CP. What we need is that after the $\mathrm{CP}$ removal, we obtain the time reversal version of only information symbols, i.e., $\zeta\left(\operatorname{FFT}\left(\mathrm{u}_{\mathrm{i}}\right)\right), \forall i \in \mathbb{Z}_{4}$. Then by using some properties of FFT/IFFT, we achieve the feasible definition as follows.

Definition 2.1 According to the processed four OFDM symbols at relay node $\mathrm{R}_{1}$ we can obtain

$\zeta\left(\varphi_{1}^{\prime}\right) \circledast \zeta\left(\mathrm{FFT}\left(\mathrm{u}_{\mathrm{i}}\right)\right)$

at destination node if we remove the $\mathrm{CP}$ as in a conventional OFDM system to get an $\mathrm{N}$-point vector and shift the last $\tau_{1}^{\prime}=1_{\mathrm{cp}}-\tau 1+1$ samples of the $\mathrm{N}$-point vector as the first $\tau_{1}^{\prime}$ samples. Here $\varphi_{1}^{\prime}$ is an $\mathrm{N} \times 1$ vector defined as

$\varphi_{1}^{\prime}=\left(\alpha_{\mathrm{s} 1}(0), \ldots, \alpha_{\mathrm{s} 1}(\mathrm{~L}-1), 0, \ldots, 0\right)$,

and $\tau_{1}^{\prime}$ denotes the maximum path delay of channel $\varphi_{1}$ from source node $S$ to relay node $R_{1}$, i.e., $\tau_{1}=$ $\max _{1}\left\{\tau_{1}, \mathrm{~s}_{1}\right\}$. In a similar way, we define another $\mathrm{N} \times 1$ vector

$\kappa_{1}^{\prime}=\left(\alpha_{\mathrm{r} 1}(0), \ldots, \alpha_{\mathrm{r} 1}(\mathrm{~L}-1), 0, \ldots, 0\right)$.

At destination node $\mathrm{D}$, after the $\mathrm{CP}$ removal, the received four successive OFDM symbols can be written as

$\mathrm{y}_{0}=\lambda\left(\sqrt{\mathrm{pt}} \zeta\left(\mathrm{FFT}\left(\mathrm{u}_{0}\right)\right) \circledast \zeta\left(\varphi_{1}^{\prime}\right)+\overline{\mathrm{n}}_{10}\right) \circledast \kappa_{1}^{\prime}+\mathrm{n}_{0}$

$\mathrm{y}_{1}=\lambda\left(\sqrt{\mathrm{ptt}} \zeta\left(\mathrm{FFT}\left(\mathrm{u}_{0}+\mathrm{u}_{2}\right)\right) \circledast \zeta\left(\varphi_{1}^{\prime}\right)+\overline{\mathrm{n}}_{10}+\overline{\mathrm{n}}_{12}\right) \circledast \kappa_{1}^{\prime}+\mathrm{n}_{1}$

$\mathrm{y}_{2}=\lambda\left(\sqrt{\mathrm{pt}}\left(\mathrm{FFT}\left(\mathrm{u}_{1}\right)\right) * \circledast \mathrm{t}_{\mathrm{dd} 2} \circledast \mathrm{t}_{1}^{\prime} \circledast \varphi_{2}^{\prime}+\overline{\mathrm{n}}_{21}^{*}\right) \circledast \kappa_{2}^{\prime}+\mathrm{n}_{2}$

$\mathrm{y}_{3}=\lambda\left(\sqrt{\mathrm{pt}}\left(\mathrm{FFT}\left(\mathrm{u}_{3}+\mathrm{u}_{1}\right)\right) * \circledast \mathrm{t}_{\mathrm{sd} 2} \circledast \mathrm{t}_{1}^{\prime} \circledast \varphi_{2}^{\prime}+\overline{\mathrm{n}}_{21}^{*}+\overline{\mathrm{n}}_{23}^{*}\right)$

$\circledast \kappa_{2}^{\prime}+\mathrm{n}_{3}$

where $t_{\mathrm{sd} 2}$ is an $\mathrm{N} \times 1$ vector that represents the timing errors in the time domain denoted as $\mathrm{t}_{\mathrm{sd} 2}=\left(0_{\tau}, \mathrm{sd} 2,1\right.$, $0, \ldots, 0)^{\mathrm{T}}$, and $0_{\tau_{\mathrm{sd} 2}}$ is a $1 \times_{\tau_{\mathrm{sd} 2}}$ vector of all zeros, and $\Gamma_{1}$ is the shift of samples in the time domain defined as $t_{1}^{\prime}=\left(O_{\tau 1}^{\prime}, 1,0, \ldots, 0\right)^{\mathrm{T}}$. Since the signals transmitted from $R_{2}$ will arrive at the destination $\tau_{\mathrm{sd} 2}$ samples later and after the $\mathrm{CP}$ removal, the signals are further shifted by $\tau_{1}$ samples. The total number of shifted samples is denoted by $\tau_{2}=\tau_{\mathrm{sd} 2}+\tau_{1}^{\prime}$. Here $\overline{\mathrm{n}}_{\mathrm{ki}}$ is the AWGN at relay node $\mathrm{R}_{\mathrm{k}}$ after the CP removal, and $\mathrm{n}_{\mathrm{i}}$ de- 
notes the AWGN at destination node D.

After that the received OFDM symbols are transformed by the N-point FFT. As mentioned before, because of the timing errors, the OFDM symbols from relay node R2 arrive at destination node $\tau_{\mathrm{sd} 2}$ samples later than that of symbols from relay node $R_{1}$. Since $1_{c p}$ is long enough, we can still maintain the orthogonality between subcarriers. The delay $\tau_{\mathrm{sd} 2}$ in the time domain corresponds to a phase change in the frequency domain, i.e., $\mathrm{f}^{\mathrm{\tau sd} 2}=\left(1, \mathrm{e}^{-12 \pi \tau \mathrm{sd} 2 / \mathrm{N}}, \ldots, \mathrm{e}^{-12 \pi \tau \mathrm{sd} 2(\mathrm{~N}-1) / \mathrm{N}}\right)^{\mathrm{T}}$,

where $\mathrm{f}=\left(1, \mathrm{e}^{-12 \pi / \mathrm{N}}, \ldots, \mathrm{e}^{-12 \pi(\mathrm{N}-1) / \mathrm{N}}\right)^{\mathrm{T}}$ and $\mathrm{\imath}=\sqrt{ }-1$. Similarly, the shift of $\tau_{1}^{\prime}$ samples in the time domain also corresponds to a phase change $\mathrm{f}_{1}^{\tau^{\prime}}$, and hence the total phase change is $\mathrm{f}^{\tau 2}$.

Denote by $\breve{y} i=\left(\breve{y}_{i 0}, \breve{y}_{i 1}, \ldots, \breve{y}_{i(N-1)}\right), \forall i \in \mathbb{Z}_{4}$, the received signals for four consecutive OFDM blocks at destination node D after the CP removal and the N-point FFT transformations. Namely, we have

$$
\begin{aligned}
& \breve{y}_{0}=\lambda\left[\sqrt{p_{t}} F F T\left(\xi\left(F F T\left(u_{0}\right)\right)\right) \circ \breve{\phi}_{1} \circ \breve{k}_{1}+\breve{\bar{n}}_{10} \circ \breve{k}_{1}\right]+\breve{\bar{n}}_{0} \\
& \breve{y}_{1}=\lambda\left[\sqrt{p_{t}} \operatorname{FFT}\left(\xi\left(F F T\left(u_{0}+u_{2}\right)\right)\right) \circ \breve{\phi}_{1} \circ \breve{k}_{1}+\left(\check{\bar{n}}_{10}+\check{\bar{n}}_{10}\right) \circ \breve{k}_{1}\right]+\check{\bar{n}}_{1} \\
& \breve{y}_{2}=\lambda\left[\sqrt{p_{t}} F F T\left(\left(F F T\left(u_{1}\right)\right) * \circ f^{\tau t} \circ \breve{\phi}_{2} \circ{\breve{k_{2}}}_{2}+\check{\bar{n}}_{21} \circ \breve{k}_{2}\right]+\check{\bar{n}}_{2}\right. \\
& \breve{y}_{3}=\lambda\left[\sqrt{p_{t}} \operatorname{FFT}\left(\left(F F T\left(u_{3}+u_{1}\right)\right) *\right) \circ f^{\tau 2} \circ \breve{\phi}_{2} \circ \breve{k}_{2}+\left(\check{\bar{n}}_{21}+\check{\bar{n}}_{23}\right) \circ \breve{k}_{2}\right]+\check{\bar{n}}_{3},
\end{aligned}
$$

Where $\breve{\phi}_{1}=F F T\left(\xi\left(\phi_{1}^{\prime}\right)\right), \quad \breve{k}_{1}=F F T\left(k_{1}^{\prime}\right), \quad \breve{\phi}_{2}=F F T\left(\left(\phi_{1}^{\prime}\right)^{*}\right), \quad \breve{k}_{2}=F F T\left(k_{2}^{\prime}\right), \quad \breve{\bar{n}}_{k i}=F F T\left(\bar{n}_{k i}\right) \quad$ and $\overline{\bar{n}}_{i}=F F T\left(\bar{n}_{i}\right), \forall k \in\{1,2)$ and $\forall i \in \mathbb{Z}_{4}$

According to the properties of the well-known FFT transforms for an $\mathrm{N} \times 1$ point vector $\mathrm{x}$, we have

$(\mathrm{FFT}(\mathrm{x})) *=\operatorname{IFFT}(\mathrm{x} *)$,

$\operatorname{FFT}(\zeta(\operatorname{FFT}(\mathrm{x})))=\operatorname{IFFT}(\operatorname{FFT}(\mathrm{x}))=\mathrm{x}$.

Therefore, the formulas in (13) can be written in the polar form on each subcarrier $\square, \forall \square \in \mathbb{Z N}$, as follows

$$
\begin{aligned}
& \left(\begin{array}{l}
y_{\mathrm{oe}} \\
y_{1 \epsilon} \\
y_{2 \epsilon} \\
y_{3 \epsilon}
\end{array}\right)=\lambda \sqrt{p_{t}}\left(\begin{array}{cccc}
\phi_{1} \hat{\kappa}_{1} & 0 & 0 & 0 \\
0 & \phi_{1} \tilde{\kappa}_{1} & 0 & 0 \\
0 & 0 & \Phi_{2} & 0 \\
0 & 0 & 0 & \Phi_{2}
\end{array}\right)\left(\begin{array}{c}
u_{0 \varepsilon} \\
u_{2 \epsilon}+u_{0 \varepsilon} \\
u_{1 e}^{*} \\
u_{3 \epsilon}^{*}+u_{1 \epsilon}^{*}
\end{array}\right)+\mathrm{c}_{0} \\
& =\lambda \sqrt{p_{t}}\left(\begin{array}{cccc}
\dot{\phi}_{1} \hat{R}_{1} & 0 & 0 & 0 \\
\dot{\phi}_{1} \kappa_{1} & \check{\phi}_{1} \tilde{\kappa}_{1} & 0 & 0 \\
0 & 0 & \Phi_{2} & 0 \\
0 & 0 & \Phi_{2} & \Phi_{2}
\end{array}\right)\left(\begin{array}{c}
u_{0 \epsilon} \\
u_{2 \epsilon} \\
u_{1 *}^{*} \\
u_{3 \epsilon}^{*}
\end{array}\right)+c_{0}
\end{aligned}
$$

which can be rewritten as

$$
\begin{aligned}
& \left(\begin{array}{l}
y_{0 \epsilon} \\
y_{1 \epsilon} \\
y_{2 \epsilon}^{*} \\
y_{3 \epsilon}^{*}
\end{array}\right)=\lambda \sqrt{p_{t}}\left(\begin{array}{cc|cc}
\phi_{1} \check{\phi}_{1} & 0 & 0 & 0 \\
\ddot{\phi}_{1} \check{\kappa}_{1} & 0 & \ddot{\phi}_{1} \check{\kappa}_{1} & 0 \\
\Phi_{2}^{*} & \Phi_{2}^{*} & 0 & 0 \\
\Phi_{2}^{*} & \Phi_{2}^{*} & \Phi_{2}^{*} & \Phi_{2}^{*}
\end{array}\right)\left(\begin{array}{l}
x_{0 \epsilon} \\
x_{1 \epsilon} \\
x_{2 \epsilon} \\
x_{3 \epsilon}
\end{array}\right)+\mathrm{e} \\
& =\mathcal{H}_{I} \mathbf{x}_{I \epsilon}+\mathcal{H}_{F} \mathbf{x}_{F \epsilon}+\mathbf{e},
\end{aligned}
$$

where $\Phi_{2} \triangleq f_{\epsilon}^{\tau_{2}} \breve{\phi}_{2} \breve{k}_{2}, \Phi_{2}^{*} \triangleq f_{\epsilon}^{\tau_{2}} \breve{\phi}_{2} \breve{k}_{2}, f_{\epsilon}^{\tau_{2}}=\exp \left(-12 \pi_{\epsilon} \tau / \mathrm{N}\right), \mathrm{H}_{\mathrm{I}}$ and $\mathrm{H}_{\mathrm{F}}$ are information generator matrix and frozen generator matrix defined, respectively, as

$\mathcal{H}_{I}=\left(\begin{array}{cc}\check{\phi}_{1} \check{\kappa}_{1} & 0 \\ \check{\phi}_{1} \check{\kappa}_{1} & 0 \\ \Phi_{2} & \Phi_{2} \\ \Phi_{2} & \Phi_{2}\end{array}\right), \quad \mathcal{H}_{F}=\left(\begin{array}{cc}0 & 0 \\ \check{\phi}_{1} \check{\kappa}_{1} & 0 \\ 0 & 0 \\ \Phi_{2} & \Phi_{2}\end{array}\right)$,

$$
\left.\begin{array}{cc}
\Phi_{2} & \Phi_{2}
\end{array}\right) \quad\left(\begin{array}{cc}
\Phi_{2} & \Phi_{2}
\end{array}\right) \quad(17)
$$

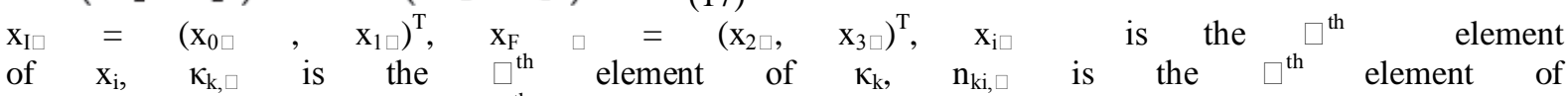
$\mathrm{n}_{\mathrm{ki}}, \quad$ and $\mathrm{n}_{\mathrm{i}, \square}$ is the $\square^{\text {th }}$ element of $\mathrm{n}_{\mathrm{i}}, \quad \forall \quad \mathrm{k}, \quad \mathrm{n}_{\mathrm{ki}, \square}$ is the $\{1,2\}$ and $\forall \quad \mathrm{i} \in$ $\mathrm{Z}_{4}$. Two $4 \times 1$ vectors $\mathrm{e}_{0}$ and $\mathrm{e}$ are the polarized noises given by $\mathrm{e}_{0}=\left(\mathrm{e}_{01}, \mathrm{e}_{02}, \mathrm{e}_{03}, \mathrm{e}_{04}\right)^{\mathrm{T}}$ and $\mathrm{e}=\left(\mathrm{e}_{01}, \mathrm{e}_{02}, \mathrm{e}^{03}, \mathrm{e}^{04}\right)^{\mathrm{T}}$, where $\mathrm{e}_{01}=\mathrm{n}_{10, \square} \kappa_{1, \square}+\check{\mathrm{n}}_{0, \square}, \mathrm{e}_{02}=\left(\mathrm{n}_{10, \square}+\mathrm{n}_{12, \square}\right) \kappa_{1, \square}+\check{\mathrm{n}}_{1, \square}, \mathrm{e}_{03}=$ $\mathrm{n}_{21, \square} \kappa_{2, \square}+\check{\mathrm{n}}_{2, \square}$ and $\mathrm{e}_{04}=\left(\mathrm{n}_{21, \square}+\mathrm{n}_{23, \square}\right) \kappa_{2, \square}+\check{\mathrm{n}}_{3, \square}$.

We note that sub-vector $\mathrm{x}_{\square} \square$ serves as the information vector while sub-vector $\mathrm{x}_{\mathrm{F}}$ as the frozen vector for the down-polarizing MIMO relay system, which can be derived from the Bhattacharyya parameter vector for the derivation of the reliability of the FSF channels, calculated in next section. The combined matrix $H=\left(\mathrm{H}_{\mathrm{I}}, \mathrm{H}_{\mathrm{F}}\right)$ has the same structure as Arikan's $4 \times 4$ polar matrix [1], [2]

$$
\mathbf{G}_{4}=\mathbf{P}_{4} \mathbf{Q}_{2}^{2}=\left(\begin{array}{llll}
1 & 0 & 0 & 0 \\
1 & 0 & 1 & 0 \\
1 & 1 & 0 & 0 \\
1 & 1 & 1 & 1
\end{array}\right),
$$


where $\mathrm{P}_{4}$ is a permutation matrix given by

$$
P_{4}=\left(\begin{array}{llll}
1 & 0 & 0 & 0 \\
0 & 0 & 1 & 0 \\
0 & 1 & 0 & 0 \\
0 & 0 & 0 & 1
\end{array}\right)
$$

\section{B. Up-polarizing MIMO-OFDM Relay System}

In the next time slot, the four consecutive OFDM blocks are processed with the up-polarizing $4 \times 4$ matrix $Q_{4}^{\prime}$ at $\mathrm{S}$, i.e.,

$$
U^{\prime}=X Q_{4}^{\prime},
$$

TABLE II

IMPLEMENTATION OF THE PF SCHEME FOR THE UP-POLARIZED SYSTEM AT RELAY NODES OF DENOTE THE $i^{\text {th }}$ OFDM BLOCK.

\begin{tabular}{|l|l|l|l|l|}
\hline & Polar $\mathrm{R}_{1}$ & Polar $\mathrm{R}_{1}$ & Process $\mathrm{R}_{1}$ & Process $\mathrm{R}_{2}$ \\
\hline $\mathrm{OF}_{0}$ & $\breve{\mathrm{r}}_{10}^{\prime}+\breve{r}_{12}^{1}$ & $\breve{r}_{20}^{\prime}$ & $\zeta\left(\breve{\mathrm{r}}_{10}^{\prime}+\breve{r}_{12}^{\prime}\right)$ & 0 \\
\hline $\mathrm{OF}_{1}$ & $\breve{r}_{11}^{\prime}$ & $\breve{\mathrm{r}}_{21}^{\prime}+\breve{r}_{23}^{\prime}$ & 0 & $\left(\breve{\mathrm{r}}_{21}^{\prime}+\breve{r}_{23}^{\prime}\right)^{*}$ \\
\hline $\mathrm{OF}_{2}$ & $\breve{r}_{12}^{\prime}$ & $\breve{r}_{22}^{\prime}$ & $\zeta\left(\breve{r}_{12}^{\prime}\right)$ & 0 \\
\hline $\mathrm{OF}_{3}$ & $\breve{r}_{13}^{\prime}$ & $\breve{r}_{23}^{\prime}$ & 0 & $\left(\breve{\mathrm{r}}_{23}^{\prime}\right)^{*}$ \\
\hline
\end{tabular}

where $U^{\prime}=\left(u_{0}^{\prime}, u_{1}^{\prime}, u_{2}^{\prime}, u_{3}^{\prime}\right)$ denotes the up-polarized matrix of size $\mathrm{N} \times 4, X=\left(x_{0}, x_{1}, x_{2}, x_{3}\right)$ denotes the initial signal matrix of size $\mathrm{N} \times 4$, the up-polarizing operation $Q_{4}^{\prime}$ is given by $Q_{4}^{\prime}=I_{2} \otimes Q_{2}^{1}$ and $Q_{2}^{1}$ is defined as

$$
Q_{2}^{1}=\left(\begin{array}{ll}
1 & 0 \\
1 & 1
\end{array}\right)
$$

Therefore, we have $\mathrm{u}_{2 \mathrm{k}-2}={ }_{\mathrm{x}_{2 \mathrm{k}-2}}+\mathrm{x}_{2 \mathrm{k}-1}$ and $\mathrm{u}_{2 \mathrm{k}-1}=$ $\mathrm{x}_{2 \mathrm{k}-1}, \forall \mathrm{k} \in\{1,2\}$.

In the OFDM modulator for the up-polarizing system, four resulting consecutive blocks are also modulated by $N$-point

FFT and are precoded by a CP with length lcp. Denote by

$\breve{u}_{i}^{\prime}, \forall \mathrm{i} \in \mathbb{Z} 4$ four consecutive OFDM symbols that consist of $\operatorname{FFT}\left(u_{i}^{\prime}\right)$ and the corresponding CP. At each relay Rk, the received noisy OFDM symbols, denoted by $\breve{r}_{i}^{\prime}$, will be polarized, processed and forwarded to destination node $\mathrm{D}$.

Define two vectors $u_{1}^{\prime}=\left(u_{0}^{T}, u_{1}^{T}\right)^{T}$ and $u_{2}^{\prime}=\left(u_{1}^{T}, u_{3}^{T}\right)^{T}$ such that

$u_{1}^{\prime}=\left(\left(x_{0}+x_{1}\right)^{T},\left(x_{2}+x_{3}\right)^{T}\right)^{T}$,

$u_{2}^{\prime}=\left(x_{1}^{T},+x_{3}^{T}\right)^{T}$.

After performing $\mathrm{N}$-point FFT onto $u_{i}^{\prime}, \forall \mathrm{i} \in \mathbb{Z} 4$

$\check{u}_{1}=\left(\operatorname{FFT}\left(x_{0}+x_{1}\right)^{\mathbf{T}}, \operatorname{FFT}\left(x_{2}+x_{3}\right)^{\mathbf{T}}\right)^{\mathbf{T}}$,

$\breve{\mathrm{u}}_{2}=\left(\operatorname{FFT}\left(\mathrm{x}_{1}\right)^{\mathrm{T}}, \operatorname{FFT}\left(\mathrm{x}_{3}\right)^{\mathrm{T}}\right)^{\mathrm{T}}$.(22)

Therefore, the received OFDM symbols at relay node $\mathrm{R}_{\mathrm{k}}$ can be written as 


$$
\begin{aligned}
& \breve{r}_{k 0}^{\prime}=\sqrt{p t} \breve{u}_{0}^{\prime} \otimes \phi_{k}+\bar{n}_{k 0}, \\
& \breve{r}_{k 1}^{\prime}=\sqrt{p t} \breve{u}_{1}^{\prime} \otimes \phi_{k}+\bar{n}_{k 1}, \\
& \breve{r}_{k 2}^{\prime}=\sqrt{p t} \breve{u}_{2}^{\prime} \otimes \phi_{k}+\bar{n}_{k 2}, \\
& \breve{r}_{k 3}^{\prime}=\sqrt{p t} \breve{u}_{3}^{\prime} \otimes \phi_{k}+\bar{n}_{k 3},
\end{aligned}
$$

Then two relay nodes polarize, process and forward the received noisy OFDM symbols as shown in Table II. After that we obtain

$$
\breve{v}_{0}^{\prime}=\zeta\left(\breve{r}_{10}^{\prime}+\breve{r}_{12}^{\prime}\right), \breve{v}_{1}^{\prime}=\zeta\left(\breve{r}_{12}^{\prime}\right), \breve{v}_{2}^{1}=\left(\breve{r}_{21}^{\prime}+\breve{r}_{23}^{\prime}\right) * \text { and } \breve{v}_{3}^{1}=\left(\breve{r}_{23}^{\prime}\right) * \text {. }
$$

After performing the processing, each relay node $R_{k}$ amplifies the yielded signals with a scalar $\lambda=\sqrt{p_{r} /\left(p_{t}+1\right)}$ and forwards them to the destination node $\mathrm{D}$.

At destination node $\mathrm{D}$, the $\mathrm{CP}$ is removed for each OFDM symbol before being depolarized to decode the initial information with high reliability. Then the received noisy OFDM symbols for four successive OFDM symbol durations can be written as

$$
\begin{aligned}
& \mathrm{y}_{0}^{\prime}=\lambda\left(\sqrt{\mathrm{p}_{\mathrm{t}}} \zeta\left(\mathrm{FFT}\left(\mathrm{u}_{0}^{\prime}+\mathrm{u}_{2}^{\prime}\right)\right)\right. \\
& \left.\otimes \zeta\left(\phi_{1}^{\prime}\right)+\overline{\mathrm{n}}_{10}+\overline{\mathrm{n}}_{20}\right) \otimes \mathrm{k}_{1}^{\prime}+\overline{\mathrm{n}}_{0} \\
& \mathrm{y}_{1}^{\prime}=\lambda\left(\sqrt{\mathrm{p}_{\mathrm{t}}} \zeta\left(\mathrm{FFT}\left(\mathrm{u}_{2}^{\prime}\right)\right) \otimes \zeta\left(\phi_{1}^{\prime}\right)\right. \\
& \left.+\overline{\mathrm{n}}_{20}\right) \otimes \mathrm{k}_{1}^{\prime}+\overline{\mathrm{n}}_{1} \\
& \mathrm{y}_{2}^{\prime}=\lambda\left(\sqrt{\mathrm{p}_{\mathrm{t}}} \zeta\left(\mathrm{FFT}\left(\mathrm{u}_{1}^{\prime}+\mathrm{u}_{3}^{\prime}\right)\right) *\right. \\
& \left.\otimes \Gamma_{\mathrm{sd} 2} \otimes \Gamma_{1}^{\prime} \otimes \phi_{2}^{\prime}+\overline{\mathrm{n}}_{21}^{*}+\overline{\mathrm{n}}_{23}^{*}\right) \otimes \mathrm{k}_{2}^{\prime}+\overline{\mathrm{n}}_{2} \\
& \mathrm{y}_{3}^{\prime}=\lambda\left(\sqrt{\mathrm{p}_{\mathrm{t}}} \zeta\left(\mathrm{FFT}\left(\mathrm{u}_{3}\right)\right) * \otimes \Gamma_{\mathrm{sd} 2}\right. \\
& \left.\otimes \Gamma_{1}^{\prime} \otimes \phi_{2}^{\prime}+\overline{\mathrm{n}}_{23}^{*}\right) \otimes \mathrm{k}_{2}^{\prime}+\overline{\mathrm{n}}_{3}
\end{aligned}
$$

Consequently, after the CP removal and N-point FFT transformations, the received four consecutive OFDM blocks can be given by

$$
\begin{aligned}
& \mathrm{y}_{0}^{\prime}=\lambda\left[\sqrt{\mathrm{p}_{\mathrm{t}}} \mathrm{FFT}\left(\zeta\left(\operatorname{FFT}\left(\mathrm{u}_{0}+\mathrm{u}_{2}\right)\right)\right) \circ \breve{\phi}_{1} \circ \breve{\mathrm{k}}_{1}\right. \\
& \left.+\left(\overline{\mathrm{n}}_{10}+\overline{\mathrm{n}}_{12}\right) \mathrm{ok}_{1}\right]+\breve{\mathrm{n}}_{0} \\
& \mathrm{y}_{1}^{\prime}=\lambda\left[\sqrt{\mathrm{p}_{\mathrm{t}}} \mathrm{FFT}\left(\zeta\left(\mathrm{FFT}\left(\mathrm{u}_{2}\right)\right)\right) \circ \breve{\phi}_{1} \circ \breve{\mathrm{k}}_{1}\right. \\
& \left.+\overline{\mathrm{n}}_{12} \mathrm{Ok}_{1}\right]+\breve{\mathrm{n}}_{1} \\
& \mathrm{y}_{2}^{\prime}=\lambda\left[\sqrt{\mathrm{p}_{\mathrm{t}}} \operatorname{FFT}\left(\zeta\left(\operatorname{FFT}\left(\mathrm{u}_{1}+\mathrm{u}_{3}\right)\right)\right) \circ f^{\mathrm{T} 2}\right. \\
& \left.\circ \breve{\phi}_{2} \circ \breve{\mathrm{k}}_{2}+\left(\overline{\mathrm{n}}_{21}^{*}+\overline{\mathrm{n}}_{23}^{*}\right) \breve{\mathrm{k}}_{2}\right]+\breve{\mathrm{n}}_{2} \\
& \mathrm{y}_{3}^{\prime}=\lambda\left[\sqrt{\mathrm{p}_{\mathrm{t}}} \operatorname{FFT}\left(\zeta\left(\operatorname{FFT}\left(\mathrm{u}_{3}\right)\right)^{*}\right) \circ f^{\mathrm{T} 2}\right. \\
& \left.\circ \breve{\phi}_{2} \circ \breve{\mathrm{k}}_{2}+\overline{\mathrm{n}}_{23}^{*} \mathrm{o \textrm {k }} 2\right]+\breve{\mathrm{n}}_{3}
\end{aligned}
$$

For each subcarrier $\square, \forall \square \in \mathbb{Z}$, the formulas in (25) can be written in the up-polarizing structure as follows,

$$
\begin{aligned}
& \left(\begin{array}{l}
y_{0 \epsilon} \\
y_{1 \epsilon} \\
y_{2 \epsilon} \\
y_{3 \epsilon}
\end{array}\right)=\lambda \sqrt{p_{t}}\left(\begin{array}{cccc}
\phi_{1} \bar{\kappa}_{1} & 0 & 0 & 0 \\
0 & \bar{\phi}_{1} \bar{\beta}_{1} & 0 & 0 \\
0 & 0 & \Phi_{2} & 0 \\
0 & 0 & 0 & \Phi_{2}
\end{array}\right)\left(\begin{array}{c}
u_{0 e}+u_{0 e} \\
u_{2 \epsilon} \\
u_{1 \epsilon}^{*}+u_{1 \epsilon}^{*} \\
u_{3 \epsilon}^{*}
\end{array}\right)+\mathbf{e}_{b}^{\prime} \\
& =\lambda \sqrt{p_{t}}\left(\begin{array}{cccc}
\Phi_{1} \tilde{K}_{1} & \dot{\phi}_{1} \dot{\kappa}_{1} & 0 & 0 \\
0 & \mathscr{\phi}_{1} \tilde{\kappa}_{1} & 0 & 0 \\
0 & 0 & \Phi_{2} & \Phi_{2} \\
0 & 0 & 0 & \Phi_{2}
\end{array}\right)\left(\begin{array}{l}
u_{0 \epsilon} \\
u_{2 e} \\
u_{1 \epsilon}^{*} \\
u_{3 e}^{*}
\end{array}\right)+\mathbf{e}_{0}^{\prime}
\end{aligned}
$$


which can be written as

$$
\left(\begin{array}{l}
y_{0 c} \\
y_{1 \epsilon} \\
y_{2 \epsilon}^{*} \\
y_{3 \epsilon}^{*}
\end{array}\right)=\lambda \sqrt{p_{t}}\left(\begin{array}{cc|cc}
\bar{\phi}_{1} \bar{\kappa}_{1} & \phi_{1} \bar{\kappa}_{1} & \bar{\phi}_{1} \bar{\kappa}_{1} & \bar{\phi}_{1} \bar{\kappa}_{1} \\
0 & 0 & \phi_{1} \bar{\kappa}_{1} & \phi_{1} \bar{\kappa}_{1} \\
0 & \Phi_{2}^{*} & 0 & \Phi_{2}^{*} \\
0 & 0 & 0 & \Phi_{2}^{*}
\end{array}\right)\left(\begin{array}{l}
x_{0 c} \\
x_{1 \epsilon} \\
x_{2 \epsilon} \\
x_{3 \epsilon}
\end{array}\right)+\mathrm{e}^{t}
$$

where $H_{I}^{\prime}$ and $H_{F}^{\prime}$ are information generator matrix and frozen generator matrix defined, respectively, as

$$
\mathcal{H}_{l}^{\prime}=\left(\begin{array}{cc}
\dot{\phi}_{1} \tilde{\kappa}_{1} & \dot{\phi}_{1} \tilde{\kappa}_{1} \\
0 & 0 \\
0 & \Phi_{2}^{*} \\
0 & 0
\end{array}\right), \mathcal{H}_{F}^{\prime}=\left(\begin{array}{cc}
\dot{\phi}_{1} \tilde{\kappa}_{1} & \dot{\phi}_{1} \tilde{\kappa}_{1} \\
\dot{\phi}_{1} \tilde{\kappa}_{1} & \dot{\phi}_{1} \tilde{\kappa}_{1} \\
0 & \Phi_{2}^{*} \\
0 & \Phi_{2}^{*}
\end{array}\right)
$$

$x_{F \in}^{\prime}=\left(x_{0 \in}, x_{1 \in}\right)^{T}$ denote the frozen vector (bits), $x_{I \in}^{\prime}=\left(x_{2 \epsilon}, x_{3 \in}\right)^{T}$ is the vector (bits), $e_{0}^{\prime}$ and $e^{\prime}=$

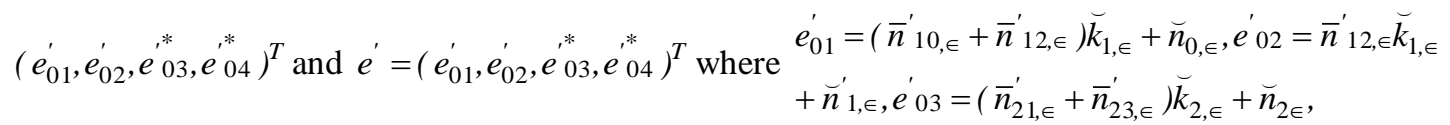

and $e^{\prime} 04=\bar{n}_{23, \epsilon}^{\prime} \breve{k}_{2, \epsilon}+\breve{n}_{1, \epsilon}$.

Next, we can design the polar MIMO-OFDM relay system by switching to polarizing four OFDM symbols for the FSF channels. Based on the down-polarizing system to transmit the $\in{ }^{\text {th }}$ subcarrier of four OFDM symbols, we have the received vector of size $4 \times 1$ given by

$y_{\in}=H_{I \times I \in}+H_{F \times F, \in}+e$,

whereas deploying the up-polarizing system, we have

$$
y_{\in}^{\prime}=H_{F, \in}^{\prime}+H_{I}^{\prime} \times_{I, \in}^{\prime}+e^{\prime}
$$

We can decode the initial information vectors $x_{I}$ and $x_{I}^{\prime}$, respectively respectively, using the conventional the ML receiver or the ZF/MMSE receiver after depolarizing the transmitted signals at destination node $\mathrm{D}$. However, in this paper we introduce a polar receiver via the polar decoding under a low complexity SIC strategy that can bring out similar performance behaviors as that of ML decoding for small number of OFDM blocks, i.e., $\mathrm{N}_{\mathrm{S}}=4$. Fortunately, due to the benefits of polar coding sequences for the large number $\mathrm{N}_{\mathrm{s}}=$ $2^{\mathrm{n}}[1]-[6]$ it also shares the good BER performance behavior of polarizing FSF channels in terms of its capacity-achieving properties [25] as non-negative integer $n$ goes to infinity.

\section{De Coding Of THe Pol Ar Mimo-Ofdm Re L Ay SystEm}

We consider all single-links of the FSF channel $\mathrm{H}$ from each pair of transmit antenna of source node $\mathrm{S}$ and receive antenna of relay node $R_{k}$, and $K$ from relay node $R_{k}$ to destination node $D$, which are independent complex Gaussian random variables with zero-mean and unit-variance. Each single-link channel, denoted by $\mathrm{W}$, has the transition probability $\mathrm{W}(\mathrm{y} \mid \mathrm{x})$, where $\mathrm{x}, \mathrm{y} \in \mathrm{A}$. As a useful measurement of the reliability of the wireless network, there is a conventional channel parameter, the symmetric capacity $\mathbf{I}(\mathrm{W})$ with some modulations [25]. We note that parameter $\mathbf{I}(\mathrm{W})$ is the highest rate at which the reliable communication is possible using inputs with equal probabilities.

Polarizations of the FSF channels are derived from the are derived from the $\mathrm{N}_{\mathrm{s}}=2^{\mathrm{n}}$ OFDM symbols polarization with generator matrix $G_{N_{2}} \in\left\{Q_{2}^{\otimes n}, Q_{2}^{\prime \otimes n}\right\}$, which is an operation by which one manufacture out of Ns independent OFDM symbols W yields a second set of Ns splitting OFDM symbols $\left\{W_{N s}^{(i)}: i \in \mathbb{Z}_{N s}\right\}$ that show a polarization effect in a sense that, as Ns becomes large, the capacity terms $\left\{I W_{N s}^{(i)}: i \in \mathbb{Z}_{N s}\right\}$ tend towards one or zero for all but a vanishing fraction of indices $i$ [1]. In this paper, we only consider polarizations of an MIMO-OFDM relay wireless system including Ns $=4$ OFDM symbols, i.e., the combination of four OFDM symbols yields a second set of four splitting FSF channels $\left\{W_{4}^{(i)}: i \in \mathbb{Z}_{4}\right\}$. This channel polarization consists of two operations, i.e., OFDM combining and OFDM splitting.

\section{OFDM Combining}

While deriving the FSF channel combining operation of the polar system with four OFDM blocks, we combine four OFDM symbols, denoted by $\mathrm{W}$, in a recursive manner to produce a multi-level structure channel $\mathrm{W}_{4}$. We consider OFDM combining and splitting for the $\epsilon^{\text {th }}$ subcarrier of each OFDM block with the downpolarizing information bits $\mathrm{x}_{I \epsilon}$ or the up-polarizing information bits $x_{I \in}^{\prime}$. Without loss of generality, we only 
consider the OFDM combining and splitting operations of the down-polarizing system with four OFDM blocks while showing the feasibility of the polar system. As for the OFDM combining and splitting operations of the up-polarizing system, we can achieve the similar results while referring to the polar coding processing in [1], [2]. Based on the down-polarizing system in (29), we define the down-combining equivalent FSF channel as $H=\left(H_{I}, H_{F}\right)$ expressed in (17), respectively. We note that the OFDM combining operations have the similar structure fashion as Arikan codes generated from $G 4=P 4 Q_{2}^{\otimes 2}$, i.e.,

$\mathrm{G}_{4}=\left(\begin{array}{llll}1 & 0 & 0 & 0 \\ 1 & 0 & 1 & 0 \\ 1 & 1 & 0 & 1 \\ 1 & 0 & 0 & 1\end{array}\right)$

It implies that the present polar system can be decoded via the depolarizing algorithm with a recursiveness feature.

Without causing undue prejudice or confusion for description, we especially take the simplified notations in this section as follows. The notation $x_{i} \triangleq x_{i, E}$ denotes the initial input signals at source node $S, u_{i} \triangleq u_{i, e}$ the polarized signals at source node $S, r_{i} \triangleq r_{i, \epsilon}$ the received signals at relay node $R, v_{i} \triangleq v_{i, \epsilon}$ the polarized signals at relay nodes $R$, and $y_{i} \triangleq y_{i, E}$ the received signals at destination node $D$, which are all corresponding to the $\epsilon^{\text {th }}$ subcarrier of the $\mathrm{i}^{\text {th }}$ OFDM symbol. For each subcarrier $\mathrm{x}_{\mathrm{i}, \square}, \mathrm{y}_{\mathrm{i}, \square}, \mathrm{u}_{\mathrm{i}, \square}$, and $\mathrm{r}_{\mathrm{i}, \square}$ of $\mathrm{x}_{\mathrm{i}}, \mathrm{y}_{\mathrm{i}}, \mathrm{u}_{\mathrm{i}}$ and $\mathrm{r}_{\mathrm{i}}$, we also take the notations $\mathrm{x} \triangleq\left(\mathrm{x}_{0, \square}, \mathrm{x}_{1, \square}, \mathrm{x}_{2, \square}, \mathrm{x}_{3, \square}\right)^{\mathrm{T}}, \mathrm{x}_{\mathrm{k}} \triangleq\left(\mathrm{x}_{2 \mathrm{k}-2, \square}, \mathrm{x}_{2 \mathrm{k}-1, \square}\right)^{\mathrm{T}}, \mathrm{y} \triangleq\left(\mathrm{y}_{0, \square}, \mathrm{y}_{1, \square}, \mathrm{y}_{2, \square}, \mathrm{y}_{3, \square}\right)^{\mathrm{T}}, \mathrm{y}_{\mathrm{k}} \triangleq\left(\mathrm{y}_{2 \mathrm{k}-2, \square}, \mathrm{y}_{2 \mathrm{k}-1, \square}\right)^{\mathrm{T}}$, $\mathrm{u}_{\mathrm{k}} \triangleq\left(\mathrm{u}_{2 \mathrm{k}-2, \square}, \mathrm{u}_{2 \mathrm{k}-1, \square}\right)^{\mathrm{T}}$, and $\mathrm{r}_{\mathrm{k}} \triangleq\left(\mathrm{r}_{2 \mathrm{k}-2, \square}, \mathrm{r}_{2 \mathrm{k}-1, \square}\right)^{\mathrm{T}}, \forall \mathrm{k} \in\{1,2\}$ and $\forall \square \in \mathbb{Z}_{\mathrm{N}}$.

According to the down-polarizing system, we derive the OFDM down-combining operation of the polar FSF channels in terms of the above simplified notations.

This process begins with the low-level of the recursion at $\mathrm{R}_{\mathrm{k}}$ that combines two independent OFDMs with transition probability W, which results in the OFDM down-combining for the second level combining FSF channel $\mathrm{W}_{2}$ for $\mathrm{R}_{\mathrm{k}}$ with transition probabilities

$\mathrm{W}_{2}\left(\mathrm{y}_{\mathrm{k}} \mid \mathrm{r}_{\mathrm{k}}\right)=\mathrm{W}\left(\mathrm{y}_{2 \mathrm{k}-2} \mid \mathrm{r}_{2 \mathrm{k}-2}\right) \cdot \mathrm{W}\left(\mathrm{y}_{2 \mathrm{k}-1} \mid \mathrm{r}_{2 \mathrm{k}-2}+\mathrm{r}_{2 \mathrm{k}-1}\right)$.

Similarly, the OFDM down-combing for the FSF channel $\mathrm{W}_{2}$ at source node $\mathrm{S}$ can be obtained with transition probabilities

$\mathrm{W}_{2}\left(\mathrm{u}_{1} \mid \mathrm{x}_{\mathrm{k}}\right)=\mathrm{W}\left(\mathrm{u}_{2 \mathrm{k}-2} \mid \mathrm{x}_{2 \mathrm{k}-2}\right) \cdot \mathrm{W}\left(\mathrm{u}_{2 \mathrm{k}-1} \mid \mathrm{x}_{2 \mathrm{k}-2}+\mathrm{x}_{2 \mathrm{k}-1}\right)$.

Furthermore, the third level of recursion for the MIMO relay system combines two independent FSF channels $\mathrm{W}_{2}$ to establish the high level FSF channel $\mathrm{W}_{4}$ with transition probabilities calculated from the recursive formula $\mathrm{W}_{4}(\mathrm{y} \mid \mathrm{x})=\mathrm{W}_{2}\left(\mathrm{y}_{0} \mid \mathrm{x}_{0}\right) \cdot \mathrm{W}_{2}\left(\mathrm{y}_{1} \mid \mathrm{x}_{0}+\mathrm{x}_{1}, \mathrm{x}_{2}+\mathrm{x}_{3}\right)$

$=\mathrm{W}\left(\mathrm{y}_{0} \mid \mathrm{x}_{0}\right) \mathrm{W}\left(\mathrm{y}_{1} \mid \mathrm{x}_{0}+\mathrm{x}_{2}\right) \mathrm{W}\left(\mathrm{y}_{2} \mid \mathrm{x}_{0}+\mathrm{x}_{1}\right) \mathrm{W}\left(\mathrm{y}_{3} \mid \sum_{i=0}^{3} x_{i}\right)$.

In the similar way, we calculate transition probabilities of OFDM up-combining for the second level FSF channel $W_{2}^{\prime}$ at $\mathrm{R}_{\mathrm{k}}$ with transition probabilities

$\mathrm{W}_{2}^{\prime}\left(y_{k}^{\prime} \mid r_{k}^{\prime}\right)=\mathrm{W}^{\prime}\left(y_{2 k-2}^{\prime} \mid r_{2 k-2}^{\prime}+r_{2 k-1}^{\prime}\right) \cdot \mathrm{W}^{\prime}\left(y_{2 k-1}^{\prime} \mid r_{2 k-1}^{\prime}\right)$,

and transition probabilities of OFDM up-combining for the FSF channel $\mathrm{W}^{\prime} 2$ at $\mathrm{S}$ as

$$
\mathrm{W}_{2}^{\prime}\left(u_{k}^{\prime} \mid x_{k}^{\prime}\right)=\mathrm{W}^{\prime}\left(u_{2 k-2}^{\prime} \mid x_{2 k-2}^{\prime}+x_{2 k-1}^{\prime}\right) \cdot \mathrm{W}^{\prime}\left(u_{2 k-1}^{\prime} \mid x_{2 k-1}^{\prime}\right) \text {. }
$$

Then the third level OFDM up-combining for the FSF channel $\mathrm{W}_{4}^{\prime}$ can be derived with transition probabilities

$$
=\mathrm{W}\left(\mathrm{y}_{0} \mid \mathrm{x}_{0}\right) \mathrm{W}\left(\mathrm{y}_{1} \mid \mathrm{x}_{0}+\mathrm{x}_{2}\right) \mathrm{W}\left(\mathrm{y}_{2} \mid \mathrm{x}_{0}+\mathrm{x}_{1}\right) \mathrm{W}\left(\mathrm{y}_{3} \mid \mathrm{W}_{4}^{\prime}\left(y^{\prime} \mid x\right)=\mathrm{W}^{\prime}\left(y_{0}^{\prime} \mid x_{0}+x_{2}\right) \mathrm{W}^{\prime}\left(y_{1}^{\prime} \mid x_{0}\right) \mathrm{W}^{\prime}\left(y_{2}^{\prime} \mid \sum_{i=0}^{3} x_{i}\right)\right.
$$

$$
\text { . } \mathrm{W}^{\prime}\left(y_{3}^{\prime} \mid x_{0}+x_{1}\right) \text {. }
$$

According to (34) and (34), for the $\epsilon^{\text {th }}$ subcarrier of each OFDM symbol, we obtain the OFDM combining for the third level FSF channels $\mathrm{W} 4$ and $\mathrm{W}_{4}^{\prime}$ with transition probabilities

$$
\begin{aligned}
& \mathrm{W}_{4}\left(\mathrm{y}_{\epsilon} \in \mathrm{x}_{\epsilon}\right)=\mathrm{W}\left(\mathrm{y}_{0}, \in \mid \mathrm{x}_{0, \epsilon}\right) \cdot \mathrm{W}\left(\mathrm{y}_{1,} \in \mathrm{x}_{0,} \in+\mathrm{x}_{2, \epsilon}\right) \\
& \mathrm{W}\left(\mathrm{y}_{2,} \in \mathrm{x}_{0}+\mathrm{x}_{1, \epsilon}\right) \cdot \mathrm{W}\left(\mathrm{y}_{3}, \epsilon \mid \sum_{i=0}^{3} x_{i}\right),
\end{aligned}
$$


$\mathrm{W}_{4}^{\prime}\left(y_{0, \epsilon}^{\prime} \mid x_{\epsilon}\right)=\mathrm{W}^{\prime}\left(y_{0, \epsilon}^{\prime} \mid x_{0}, \epsilon+x_{2}\right) \cdot \mathrm{W}^{\prime}\left(y_{1, \epsilon}^{\prime} \mid x_{0}\right)$

. W' $\left(y_{2, \epsilon}^{\prime} \mid \sum_{i=0}^{3} x_{i, \epsilon}\right) \cdot \mathrm{W}^{\prime}\left(y_{3, \in}^{\prime} \mid x_{0}+x_{1, \in)}\right.$.

OFDM Splitting

Next, we consider the OFDM down-splitting operation for the down-polarizing system, which splits the synthesized FSF channel $\mathrm{W}_{4}$ back into a set of equivalent single-link FSF channels $\mathrm{W}_{4}^{(i)}, \forall i \in \mathbb{Z} 4$. The downsplitting OFDMs can be used for the transmission of signals in the polar system with high reliability in terms of transition probabilities, as well as down-splitting channel capacity $I\left(\mathrm{~W}_{4}^{(i)}\right)$ [25]. At each relay node $\mathrm{R}_{\mathrm{k}}$, we define the OFDM down-splitting operations as one-one maps that illustrate the relation of the transition probabilities of each subcarrier of two level OFDM downcombining FSF channels $\mathrm{W}$ and $\mathrm{W}_{2}$ as follows

$\mathrm{W}_{2}^{(0)}\left(y_{k} \mid r_{2 k-2}\right)=\sum \frac{1}{2} \mathrm{~W}\left(y_{2 k-2} \mid r_{2 k-2}\right) \mathrm{W}\left(y_{2 k-1} \mid r_{2 k-2}+r_{2 k-1}\right)$;

$\mathrm{W}_{2}^{(1)}\left(y_{k}, r_{2 k-2} \mid r_{2 k-1}\right)=\frac{1}{2} \mathrm{~W}\left(y_{2 k-1} \mid r_{2 k-2}+r_{2 k-1}\right)$.

Considering all nodes $\mathrm{S}, \mathrm{R}$ and $\mathrm{D}$ for the whole polar system, for any subcarrier we derive the high-level OFDM downsplitting operations with the transition probabilities given by

$$
\begin{aligned}
& \mathrm{W}_{4}^{(0)}\left(y \mid x_{0}\right)=\sum_{x_{1}} \frac{1}{8} \mathrm{~W}_{2}^{(0)}\left(y_{1} \mid x_{0}\right) \mathrm{W}_{2}^{(0)}\left(y_{2} \mid x_{0}+x_{1}\right), \\
& \mathrm{W}_{4}^{(1)}\left(y, x_{0} \mid x_{1}\right)=\frac{1}{8} \mathrm{~W}_{2}^{(0)}\left(y_{1} \mid x_{0}\right) \mathrm{W}_{2}^{(0)}\left(y_{2} \mid x_{0}+x_{1}\right), \\
& \mathrm{W}_{4}^{(2)}\left(y, x_{1} \mid x_{2}\right)=\sum_{x_{3}} \frac{1}{8} \mathrm{~W}_{2}^{(1)}\left(y_{1}, x_{0} \mid x_{2}\right) \\
& \cdot \mathrm{W}_{2}^{(1)}\left(y_{2}, x_{0}+x_{1} \mid x_{2}+x_{3}\right), \\
& \mathrm{W}_{4}^{(3)}\left(y, x_{1}, x_{2} \mid x_{3}\right)=\frac{1}{8} \mathrm{~W}_{2}^{(1)}\left(y_{1}, x_{0} \mid x_{2}\right) \\
& \cdot \mathrm{W}_{2}^{(1)}\left(y_{2}, x_{0}+x_{1} \mid x_{2}+x_{3}\right) .
\end{aligned}
$$

Namely, for the $\epsilon^{\text {th }}$ subcarrier of each OFDM symbol we achieve the transition probabilities as follows

$$
\begin{aligned}
\mathrm{W}_{4}^{(0)}\left(y_{\epsilon} \mid x_{0, \epsilon}\right)=\sum_{x_{1, \epsilon}} \frac{1}{8} \mathrm{~W}_{2}^{(0)}\left(y_{1, \epsilon} \mid x_{0, \epsilon}\right) \mathrm{W}_{2}^{(0)}\left(y_{2, \epsilon} \mid x_{0, \epsilon}+x_{1, \epsilon}\right), \\
\mathrm{W}_{4}^{(1)}\left(y_{\epsilon}, x_{0, \epsilon} \mid x_{1, \epsilon}\right)=\frac{1}{8} \mathrm{~W}_{2}^{(0)}\left(y_{1, \epsilon} \mid x_{0, \epsilon}\right) \mathrm{W}_{2}^{(0)}\left(y_{2, \epsilon} \mid x_{0, \epsilon}+x_{1, \epsilon}\right), \\
\mathrm{W}_{4}^{(2)}\left(y_{\epsilon}, x_{1, \epsilon} \mid x_{2, \epsilon}\right)=\sum_{x_{3, \epsilon}} \frac{1}{8} \mathrm{~W}_{2}^{(1)}\left(y_{1, \epsilon}, x_{0, \epsilon} \mid x_{2, \epsilon}\right) \\
\cdot \cdot \mathrm{W}_{2}^{(1)}\left(y_{2, \epsilon}, x_{0, \epsilon}+x_{1, \epsilon} \mid x_{2, \epsilon}+x_{3, \epsilon}\right), \\
\mathrm{W}_{4}^{(3)}\left(y_{, \in}, x_{1, \epsilon}, x_{2, \epsilon} \mid x_{3, \epsilon}\right)=\frac{1}{8} \mathrm{~W}_{2}^{(1)}\left(y_{1, \epsilon}, x \mid x_{2, \epsilon}\right) \\
\cdot \mathrm{W}_{2}^{(1)}\left(y_{2, \epsilon}, x_{0, \epsilon}+x_{1, \epsilon} \mid x_{2, \epsilon}+x_{3, \epsilon}\right) .
\end{aligned}
$$

Similarly, the OFDM up-splitting operation that illustrates the relation of OFDM up-combining for two FSF channels $\mathrm{W}^{\prime}$ and $W_{2}^{\prime}$ is

$$
\begin{aligned}
& \mathrm{W}_{2}^{(0)}\left(y_{k}^{\prime} \mid r_{2 k-2}^{\prime}\right)=\sum_{r_{2 k-2}^{\prime}} \frac{1}{2} \mathrm{~W}^{\prime}\left(y_{2 k-2}^{\prime} \mid r_{2 k-2}^{\prime}+r_{2 k-1}^{\prime}\right) \mathrm{W}^{\prime}\left(y_{2 k-1}^{\prime} \mid r_{2 k-1}^{\prime}\right), \\
& \mathrm{W}_{2}^{(1)}\left(y_{k}^{\prime}, r_{2 k-2}^{\prime} \mid r_{2 k-1}^{\prime}\right)=\frac{1}{2} \mathrm{~W}^{\prime}\left(y_{2 k-2}^{\prime} \mid r_{2 k-2}^{\prime}+r_{2 k-1}^{\prime}\right) \mathrm{W}^{\prime}\left(y_{2 k-1}^{\prime} \mid r_{2 k-1}^{\prime}\right) .
\end{aligned}
$$

and the high-level OFDM up-splitting operations that illustrates the relation of two OFDM up-combining for the FSF channels $W_{2}^{\prime}$ and $W_{4}^{\prime}$ is given by

$$
\begin{aligned}
& \mathrm{W}_{4}^{(0)}\left(y^{\prime} \mid x_{0}\right)=\sum_{x_{1}} \frac{1}{8} \mathrm{~W}_{2}^{(0)}\left(y_{1}^{\prime} \mid x_{0}^{\prime}+x_{1}\right) \mathrm{W}_{2}^{(0)}\left(y_{2}^{\prime} \mid x_{1}\right), \\
& \mathrm{W}_{4}^{(1)}\left(y^{\prime}, x_{0} \mid x_{1}\right)= \frac{1}{8} \mathrm{~W}_{2}^{(0)}\left(y_{1}^{\prime} \mid x_{0}^{\prime}+x_{1}\right) \mathrm{W}_{2}^{(0)}\left(y_{2}^{\prime} \mid x_{1}\right), \\
& \mathrm{W}_{4}^{(2)}\left(y^{\prime}, x_{1} \mid x_{2}\right)= \sum_{x_{3}} \frac{1}{8} \mathrm{~W}_{2}^{(1)}\left(y_{1}^{\prime}, x_{0}+x_{1} \mid x_{2}^{\prime}+x_{3}\right) \\
& \cdot \mathrm{W}_{2}^{(1)}\left(y_{2}, x_{1} \mid x_{3}\right),
\end{aligned}
$$




$$
\begin{gathered}
\mathrm{W}_{4}^{(3)}\left(y^{\prime}, x_{1}, x_{2} \mid x_{3}\right)=\frac{1}{8} \mathrm{~W}_{2}^{(1)}\left(y_{1}^{\prime}, x_{0}+x_{1} \mid x_{2}^{\prime}+x_{3}\right) \\
\cdot \mathrm{W}_{2}^{(1)}\left(y_{2}^{\prime}, x_{1} \mid x_{3}\right) .
\end{gathered}
$$

So far we have established the polar system based on the polarization of OFDMs. It is known that the channel capacity of OFDM splitting for the FSF channel $W_{4}^{(i)}$ can be bounded by

$$
I\left(W_{4}^{(i)}\right) \leq 1-z\left(W_{4}^{(i)}\right),
$$

where $z\left(W_{4}^{(i)}\right)$ are the Bhattacharyya parameters [1] given by

$$
z\left(W_{4}^{(i)}\right)=\sum_{y_{\epsilon} \in A^{4} x_{0, \epsilon} \cdots x_{i-1, \epsilon} \in A^{i}} \sum_{x_{\epsilon} \in A} W\left(y_{\in}, x_{0, \epsilon} \cdots x_{i-1, \epsilon} \mid x_{\epsilon}\right) .
$$

Next, we analyze the reliability of the OFDM down-splitting for the FSF channels with transmission probabilities $W_{4}^{(i)}$ in

(41) based on the Bhattacharyya parameter vector

$$
\mathrm{z}_{4}=\left(\mathrm{z}_{4,0}, \mathrm{z}_{4,1}, \mathrm{z}_{4,2}, \mathrm{z}_{4,3}\right) \text {, }
$$

which can be calculated from the recursion formula [1], [2],

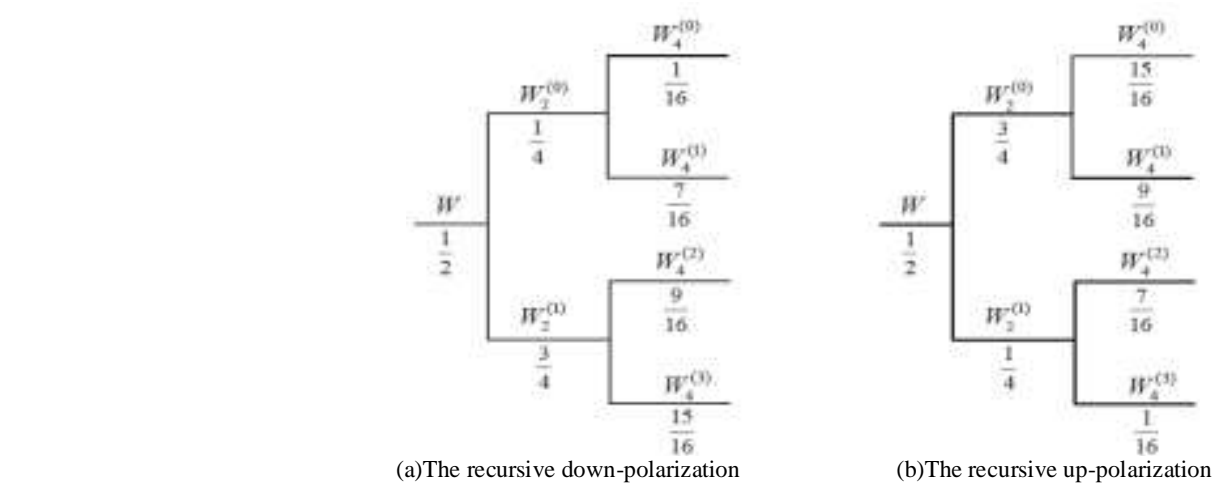

i.e.,

Fig. 2. The tree process of the Bhattacharyya parameters for the recursive polarizing OFDMs

$$
z_{2 k, j}=\left\{\begin{array}{lr}
z_{k, j}^{2}, & \text { for } 0 \leq \mathrm{j} \leq \mathrm{k}-1 \\
2 z_{k, j-k}-z_{k, j-k}^{2}, & \text { for } \mathrm{k} \leq \mathrm{j} \leq 2 \mathrm{k}-1,
\end{array}\right.
$$

for $\forall \mathrm{k} \in\{1,2\}$ starting with $\mathrm{z} 1,0=1 / 2$, shown in Fig.3(a).

From scratch, we form a permutation $\pi_{4}=\left(\mathrm{i}_{0}, \mathrm{i}_{1}, \mathrm{i}_{2}, \mathrm{i}_{3}\right)$ of

$(0,1,2,3)$ corresponding to entries of $\mathrm{x}=\left(\mathrm{x}_{0}, \mathrm{x}_{1}, \mathrm{x}_{2}, \mathrm{x}_{3}\right)^{\mathrm{T}}$ so that the inequality $\mathrm{z}_{4, \mathrm{jj}} \leq \mathrm{z}_{4, \mathrm{ik}}, \forall 0 \leq \mathrm{j}<\mathrm{k} \leq 3$, is true. Thus we have the reliability of OFDM splitting for the FSF channels given by

$$
\mathrm{z}(4)=(1 / 16,7 / 16,9 / 16,15 / 16)
$$

which creates a permutation $\pi 4=(0,1,2,3)$. It implies that for each subcarrier of the source OFDM symbols $\mathrm{x}_{\epsilon}$, the first two signals $\{\mathrm{x} 0, \epsilon, \mathrm{x} 1, \epsilon\}$ can be transmitted with higher reliability than that of the last two signals $\left\{\mathrm{x}_{2, \epsilon}\right.$, $\left.\mathrm{x}_{3, \epsilon}\right\}$, as shown in (44). Therefore, for the reliable transmission of signals while polarizing the FSF channels, we let $\left\{\mathrm{x}_{0, \epsilon}, \mathrm{x}_{1, \epsilon}\right\}$ to be the information bits that are required to be transmitted from relay nodes, and $\left\{\mathrm{x}_{2, \epsilon}, \mathrm{x}_{3, \epsilon}\right\}$ to be frozen bits that provide assistance for transmissions. In practice, the frozen bits $\left\{\mathrm{x}_{2, \epsilon}, \mathrm{x}_{3, \epsilon}\right\}$ are always be set zeros for the depolarizing for convenience, i.e., $\left\{\mathrm{x}_{2, \epsilon}=0, \mathrm{x}_{3, \epsilon}=0\right\}$. This property can be utilized for the flexible transmission of signals on the FSF channels with high reliability [1].

In the similar way, we can derive the reliability of upsplitting system for the FSF channel $W_{4}^{(i)}$ with transmission probabilities in (42) based on the Bhattacharyya parameter vector

$$
\mathrm{z}_{4}^{\prime}=\left(z_{4,0}^{\prime}, z_{4,1}^{\prime}, z_{4,2}^{\prime}, z_{4,3}^{\prime}\right) \text {, }
$$

which can be calculated from [1], [2], i.e.,

$$
z_{2 k, j}^{\prime}= \begin{cases}2 z_{k, j-k}^{\prime}-z_{k, j-k}^{\prime 2}, & \text { for } 0 \leq \mathrm{j} \leq \mathrm{k}-1 ; \\ z_{k, j}^{\prime 2}, & \text { for } \mathrm{k} \leq \mathrm{j} \leq 2 \mathrm{k}-1,\end{cases}
$$

for $\forall \mathrm{k} \in\{1,2\}$ starting with $\mathrm{z}_{1,0}=1 / 2$, shown in Fig.3(b). Consequently, we form a permutation $\pi_{4}=\left(\mathrm{i}_{0}, \mathrm{i}_{1}, \mathrm{i}_{2}\right.$, $\left.\mathrm{i}_{3}\right)$ of $(0,1,2,3)$ corresponding to entries of $\mathrm{x}=\left(\mathrm{x}_{0, \epsilon}, \mathrm{x}_{1, \epsilon}, \mathrm{x}_{2, \epsilon}, \mathrm{x}_{3, \epsilon}\right) \mathrm{T}$ so that the inequality $z_{i, j}^{i 4} \leq z_{i, k}^{i 4} \forall 0 \leq \mathrm{j}<$ $\mathrm{k} \leq 3$, is true. The reliability of OFDM splitting for the FSF channels can be derived as 


$$
\mathrm{z}(4)=(15 / 16,9 / 16,7 / 16,1 / 16)
$$

which creates a permutation $\pi_{4}=(3,2,1,0)$. It implies that for $\mathrm{x}_{\epsilon}$ embedded in four OFDM symbols, the last two signals $\left\{\mathrm{x}_{2, \epsilon}, \mathrm{x}_{3, \epsilon}\right\}$ can be transmitted with higher reliability than that of the first two signals $\left\{\mathrm{x}_{0, \epsilon}, \mathrm{x}_{1, \epsilon}\right\}$. Therefore, for the reliable transmission of signals over each subcarrier for uppolarizing system, we let $\left\{\mathrm{x}_{2, \epsilon}, \mathrm{x}_{3, \epsilon}\right\}$ to be the information bits that are required to transmit from relay nodes, whereas $\left\{\mathrm{x}_{0, \epsilon}, \mathrm{x}_{1, \epsilon}\right\}$ are frozen bits that provide assistance for the reliable transmissions.

C. The Switching Polar Relay Communications with Space-

Time-Frequency Codes

In what follows, we propose a high-reliable MIMO-OFDM relay system by rearranging a class of space-time-frequency code (STF) codes for four OFDM symbols over the FSF channels with the structure expressed in the stacked Alamouti code and the Jafarkhani code. Generally speaking, it is not difficult in practice to provide parallel transitions for an MIMO-OFDM communication system, especially for system with a large number of OFDM symbols. Therefore, a suitable STF may adapt itself to the transmission of multiple OFDM symbols with parallel transitions in the polar relay system.

According to the reliability of the switching polar relay system while calculating the Bhattacharyya parameters expressed in (44) and (46), for each subcarrier we obtain information bits $\mathrm{X}_{\mathrm{I}, \epsilon}$ and $X_{I \in}^{\prime}$ and frozen matrices $X_{\mathrm{F}, \in}$ and $X_{F \in}^{\prime}$ in the downpolarizing system and the up-polarizing system, respectively.

1) Switching Polar System with the Alamouti code: According to the above-mentioned OFDM polarizing for the FSF channels in two polar systems, i.e., the down-polarizing system and the up-polarizing system, we assume the transformed OFDM symbols $\mathrm{u}_{\mathrm{i}}$ can be encoded with the orthogonal block code that combines spatial, temporal and multipath processing for grouping signals. Actually, we can construct the Alamouti code structure on each subcarrier if the length of the $\mathrm{CP} 1_{\mathrm{cp}}$ satisfies the constraints

$$
l c p \geq_{k, l}^{\max }\left\{\tau_{l, s k}+\tau_{l, r k}+\tau_{s d 2}\right\} .
$$

To make the transmission processing clear, we consider an MIMO-OFDM relay system with one transmit antenna at source node $\mathrm{S}$, each relay node $\mathrm{Rk}$, and one receive antenna at each relay node Rk with an OFDM symbol matrix of size $4 \mathrm{~N} \times 2$ in two time slots given by the stacked Alamouti code,

$$
\Lambda(\mathrm{x})=\left(\mathrm{c}_{0}, \mathrm{c}_{1}\right)=\left(\begin{array}{l}
A\left(x_{0}, x_{1}\right) \\
A\left(x_{2}, x_{3}\right)
\end{array}\right)=\left(\begin{array}{c}
x_{0}-x_{1}^{*} \\
x_{1}-x_{0}^{*} \\
x_{2}-x_{3}^{*} \\
x_{3} x_{2}^{*}
\end{array}\right),
$$

where $\mathrm{A}\left(\mathrm{x}_{2 \mathrm{k}-2}, \mathrm{x}_{2 \mathrm{k}-1}\right), \forall \mathrm{k} \in\{1,2\}$, denotes the stacked Alamouti code [7] with $\mathrm{N}$ pairs of variables

$$
\left\{\left(\mathrm{x}_{2 \mathrm{k}-2, \epsilon}, \mathrm{x}_{2 \mathrm{k}-1), \epsilon}: \in \mathbb{Z}_{\mathrm{N}}\right\} .\right.
$$

For each subcarrier, $\forall \in \mathbb{Z}_{\mathrm{N}}$, it is given by

$$
\mathrm{A}\left(\mathrm{x}_{2 \mathrm{k}-2, \epsilon}, \mathrm{x}_{2 \mathrm{k}-1, \epsilon}\right)=\left(\begin{array}{cc}
x_{2 k-2, \epsilon} & -x_{2 k-2, \epsilon}^{*} \\
x_{2 k-1, \epsilon} & x_{2 k-1, \epsilon}^{*}
\end{array}\right) .
$$

Next, we describe the polar MIMO-OFDM relay system by switching to polarizing OFDM symbols with the stacked Alamouti code. Based on the down-polarizing system in (29) to transmit $\Lambda$ (x) expressed in (47), we have the received matrix of size $4 \times 2$ in two time slots given by

$$
\mathrm{Y}=\mathrm{H}_{\mathrm{I}} \mathrm{X}_{\mathrm{I}}+\mathrm{H}_{\mathrm{F}} \mathrm{X}_{\mathrm{F}}+\mathrm{E}
$$

where and $\mathrm{E}=(\mathrm{e}, \mathrm{e})$ is an equivalent $\mathrm{AWGN}$ vector of size $4 \times 2$. While deploying the up-polarizing system in (30) to transmit the same $\Lambda(\mathrm{x})$ in (47), we have the received matrix of size $4 \times 2$ in the next two time slots given by

$$
\mathrm{Y}^{\prime}=\mathrm{H}_{\mathrm{F}}^{\prime} \mathrm{X}_{\mathrm{F}}^{\prime}+\mathrm{H}_{\mathrm{I}}^{\prime} \mathrm{X}_{\mathrm{I}}^{\prime}+\mathrm{E}^{\prime},
$$

where $\mathrm{E}^{\prime}=\left(\mathrm{e}^{\prime}, \mathrm{e}^{\prime}\right)$ is an equivalent AWGN matrix of size $4 \times 2$ for the up-polarizing system in (30).

The two column vectors $\mathrm{c}_{0}$ and $\mathrm{c}_{1}$ in (47) are modulated for four OFDM symbols and transmitted, respectively, in the first two time slots for the down-polarizing system. Therefore, information matrix $\mathrm{X}_{\mathrm{I} \epsilon}=\mathrm{A}\left(\mathrm{x}_{0} \epsilon\right.$, $\left.\mathrm{x}_{1} \epsilon\right)$ and frozen matrix $\mathrm{X}_{\mathrm{F} \epsilon}=\mathrm{A}\left(\mathrm{x}_{2} \epsilon, \mathrm{x}_{3} \epsilon\right)$, and hence extensive information matrix $\mathrm{X}_{\mathrm{I}}=\mathrm{A}\left(\mathrm{x}_{0}, \mid \mathrm{x}_{1}\right)$ and frozen matrix $\mathrm{X}_{\mathrm{F}}=\mathrm{A}\left(\mathrm{x}_{2}, \mathrm{x}_{3}\right)$ are embedded in two OFDM symbols for two time slots, respectively. In the next two time 
slots, we switch to the uppolarizing system and achieve frozen matrix $\mathrm{X}_{\mathrm{F}}^{\prime}=\mathrm{A}\left(\mathrm{x}_{0}, \mathrm{x}_{1}\right)$ and information matrix $\mathrm{X}_{\mathrm{I}}^{\prime}$ $=\mathrm{A}\left(\mathrm{x}_{2}, \mathrm{x}_{3}\right)$ in the same two respective OFDM blocks as that of the down-polarizing system. The selections of information matrices $\left\{\mathrm{X}_{\mathrm{I}}, \mathrm{X}_{\mathrm{I}}^{\prime}\right\}$ and frozen matrices $\left\{\mathrm{X}_{\mathrm{F}}, \mathrm{X}_{\mathrm{F}}^{\prime}\right\}$ for switching polar system are based on the calculation of the Bhattacharyya parameters, as shown in Fig.2.

Theorem 3.1: According to the down-polarizing system in (49) for the first two time slots, we consider $4 N$ signals for four OFDM symbols $\left(\mathrm{x}_{0}, \mathrm{x}_{1}, \mathrm{x}_{2}, \mathrm{x}_{3}\right)$ embedded in the stacked Alamouti code in (47) for the transmission and switch to the up-polarizing system in (50) for the next two time slots. After being processed with the afore-mentioned transformations, the CP removal and the N-point FFT operations while switching the downpolarizing to up-polarizing system for four OFDM blocks in four time slots, the information matrix can be depolarized and hence be decoded with high reliability at destination node D as follows

$$
\hat{\Lambda} A(\mathrm{x})=\left(\begin{array}{ll}
X_{I} & 0_{2 \times 2} \\
0_{2 \times 2} & X_{I}^{\prime}
\end{array}\right)=\left(\begin{array}{ll}
A\left(x_{0}, x_{1}\right) & 0_{2 \times 2} \\
0_{2 \times 2} & A\left(x_{2}, x_{3}\right)
\end{array}\right),
$$

which is an orthogonal code that can achieve the full diversity.

The proof of Theorem 3.1 can be found in Appendix A.

According to the reliability of OFDM splitting for the FSF channels in (44) and (46), we implement the PF relay scheme while transmitting the information bits with four OFDM symbols via two polar systems, i.e., downpolarizing system and up-polarizing system. The polar system that are composed of down-polarizing system in (49) and up-polarizing system in (50) with the Alamouti code structure in terms of spatial, temporal and multipath for the FSF channels in four time slots has the similar performance behaviors as that of the Alamouti code for the space-time or space-frequency transmissions.

2) Switching Polar System with the Jafarkhani code: We consider a $4 \times 4$ matrix given by the Jafarkhani structure [8]

$$
\left.\mathrm{J}(\mathrm{x})=C_{0}, C_{1}, C_{2}, C_{3}\right)=\left(\begin{array}{cccc}
x_{0} & -x_{1}^{*} & -x_{2}^{*} & x_{3} \\
x_{1} & x_{0}^{*} & -x_{3}^{*} & -x_{2} \\
x_{2} & -x_{3}^{*} & x_{0} & -x_{1}^{*} \\
x_{3} & x_{2}^{*} & x_{1} & x_{0}^{*}
\end{array}\right) .
$$

For the PF scheme, two column vectors $\mathrm{c}_{0}$ and $\mathrm{c}_{2}$ are transmitted in the first time slot and the third time slot for the down-polarizing system, whereas other two column vectors $c_{1}$ and $c_{3}$ are in the second time slot and the fourth time slot for the up-polarizing system.

In the down-polarizing system at source node $\mathrm{S}$ for four successive OFDM symbols we take the notations

$$
\mathrm{X}(\mathrm{x})=\left(c_{0}, c_{2}\right)=\left(\begin{array}{l}
X_{I} \\
X_{F}
\end{array}\right),
$$

where $\mathrm{X}_{\mathrm{I}}$ and $\mathrm{X}_{\mathrm{F}}$ are information matrix and frozen matrix given by

$$
X_{I}=\left(\begin{array}{ll}
x_{0} & -x_{2}^{*} \\
x_{1} & -x_{3}^{*}
\end{array}\right), X_{F}=\left(\begin{array}{ll}
x_{2} & -x_{0}^{*} \\
x_{3} & -x_{1}^{*}
\end{array}\right) .
$$

In the up-polarizing system we use the notation

$$
\mathrm{X}^{\prime}(\mathrm{x})=\left(c_{1}, c_{3}\right)=\left(\begin{array}{c}
X_{I}^{\prime} \\
X_{F}^{\prime}
\end{array}\right),
$$

where $\mathrm{X}_{\mathrm{F}}^{\prime}$ and $\mathrm{X}_{\mathrm{I}}^{\prime}$ are frozen matrix and information matrix given, respectively, by

$$
X_{F}^{\prime}=\left(\begin{array}{cc}
-x_{1}^{*} & x_{3} \\
x_{0}^{*} & -x_{2}
\end{array}\right), X_{F}=\left(\begin{array}{cc}
-x_{3}^{*} & -x_{1}^{*} \\
x_{2}^{*} & x_{0}^{*}
\end{array}\right) .
$$

Based on the above-mentioned matrices $\mathrm{X}$ and $\mathrm{X}$, we switch to polarizing MIMO-OFDM relay system in four time slots, i.e., the down-polarizing system for transmitting $\mathrm{X}$ and the up-polarizing system for transmitting $\mathrm{X}^{\prime}$. After being depolarized and decoded at destination node $\mathrm{D}$, we achieve

$$
\hat{\mathbf{J}}(\mathrm{x})=\left(\begin{array}{cccc}
x_{0} & 0 & -x_{2}^{*} & 0 \\
x_{1} & 0 & -x_{3}^{*} & 0 \\
0 & -x_{3}^{*} & x_{0} & -x_{1}^{*} \\
0 & x_{2}^{*} & 0 & x_{0}^{*}
\end{array}\right) .
$$


Theorem 3.2: We consider $4 N$ signals for four OFDM symbols $\left(\mathrm{x}_{0}, \mathrm{x}_{1}, \mathrm{x}_{2}, \mathrm{x}_{3}\right)$ embedded in (52) for the transmission in both down-polarizing system and up-polarizing system. In the first and third time slots we transmit $\mathrm{X}$ in (53) for the down-polarizing system in (29), and switch to transmitting $\mathrm{X}^{\prime}$ in (55) in the second and fourth time slots for the up-polarizing system in (30). After being processed with abovementioned processes, the CP removal and the $N$-point FFT operations while switching the down-polarizing system to up-polarizing system for four OFDM blocks in four time slots, the received noisy information matrix can be depolarized and decoded with high reliability at destination node $D$, namely we can achieve the transmitted matrix of size $4 \times 4$ given by decision matrix $\hat{J}(x)$ expressed in (57), which is a quasi-orthogonal code equivalent to the Jafarkhani code.

The proof of Theorem 3.2 can be found in Appendix B.

We can decode the initial signal vectors $X_{I}$ and $X_{I}^{\prime}$ from $X_{I}$ and $X_{I}^{\prime}$, respectively, using the conventional Alamouti decoding or the Jafarkhani decoding with the ML receiver or the ZF/MMSE receiver after depolarizing the transmitted signals at destination node $D$. However, in this paper we introduce a polar receiver via the polar decoding under a low complexity SIC decoding strategy that can bring out similar performance behaviors as that of ML decoding. According to the reliability of OFDM splitting for the FSF channels in (44) and (46), we implement the PF relay scheme while transmitting the information bits with four OFDM symbols via switching to down-polarizing communication and up-polarizing communication, which has the similar performance behaviors as that of the Alamouti code and the Jafarkhani code.

It is shown in both (51) and (57) that the present PF MIMO-OFDM relay scheme with the STF code can achieve the similar diversity gain as that of the Alamouti code and the Jafarkhani code with OFDM polarizing for the FSF channels. According to Arikan's statement, we should obtain the good BER performance as long as the employed system is provided with a large number of OFDM symbols while implementing the polarizing operations on source node $S$ and relay nodes $R$ over the FSF channels.

\section{OFDM Depolarizing}

In this subsection, we consider the SIC decoding for the proposed polar MIMO-OFDM relay system with standard complex constellations, such as binary phase shift keying (BPSK) modulation constellation. Recall that each signal $\mathrm{x}_{\mathrm{i}, \epsilon}, \forall \in \in \mathbb{Z}_{\mathrm{N}}$, in the $\in^{\text {th }}$ subcarrier of OFDM block $\mathrm{x}_{\mathrm{i}}, \forall \mathrm{i} \in \mathbb{Z}_{4}$, is independently transmitted across $R_{k}$ and a channel output $\mathrm{y}_{\mathrm{i}, \epsilon}$ is obtained with transition probability $\mathrm{W}\left(\mathrm{y}_{\mathrm{i}, \epsilon} \mid \mathrm{x}_{\mathrm{i}, \epsilon}\right)$. For each subcarrier in four OFDM symbols we misuse the simplified notations $\mathrm{x} \triangleq \mathrm{x}_{\epsilon}=\left(\mathrm{x}_{I, \epsilon}^{T}, \mathrm{x}_{F, \epsilon}^{T}\right)^{T}, \mathrm{y} \mid \triangleq \mathrm{y}_{\epsilon}=\left(\mathrm{y}_{1, \epsilon}^{T}, \mathrm{y}_{2, \epsilon}^{T}\right)^{T}$ for the downpolarizing system, where $\mathrm{y}_{1} \triangleq \mathrm{y}_{1, \epsilon}=\left(\mathrm{y}_{0, \epsilon}, \mathrm{y}_{1, \epsilon}\right)^{\mathrm{T}}$ and $\mathrm{y}_{2} \triangleq \mathrm{y}_{2, \epsilon}=\left(\mathrm{y}_{2, \epsilon}, \mathrm{y}_{3, \epsilon}\right)^{\mathrm{T}}$. Similarly, we define $\mathrm{x}^{\prime} \triangleq x_{\in}^{\prime}=\left(\mathrm{x}_{I, \in, \mathrm{X}_{F, \in}^{T}}^{T}\right)^{T}$ and $y^{\prime} \triangleq y_{\epsilon}^{\prime}=\left(\mathrm{y}_{1, \epsilon}^{T}, \mathrm{y}_{2, \epsilon}^{T}\right)^{T}$ for each subcarrier in the up-polarizing system.

The SIC decoder of the down-polarizing system observes y and generates an estimate of $\hat{x}$ of $\mathrm{x}$. We may visualize the decoder as consisting of four decision elements for four respective OFDM symbols, each element $\hat{x}_{i}$ for source element $\hat{x}_{i}, \forall \mathrm{i} \in \mathbb{Z}_{4}$.

The OFDM depolarizing algorithm of the polar system begins with the $i^{\text {th }}$ decision element $\hat{x}_{i}$ for the downpolarizing system. It waits till receiving all previous decisions $\hat{x}_{i-1}$, and upon receiving them; it calculates the likelihood ratio (LR) $L_{4}^{i}$ as follows

$$
L_{4}^{i}\left(y, \hat{x}_{1} \cdots \hat{x}_{1-1}\right) \triangleq \frac{W_{4}^{(i)}\left(y, \hat{x}_{1} \cdots \hat{x}_{i-1} \mid 0\right)}{W_{4}^{(i)}\left(y, \hat{x}_{1} \cdots \hat{x}_{i-1} \mid 1\right)},
$$

and generates its decision as

$$
\hat{x}_{i}=\left\{\begin{array}{l}
0 \text { if } L_{4}^{(i)}\left(y, \hat{x}_{1}, \cdots . \hat{x}_{i-1}\right) \geq 1 ; \\
1 \text { otherwise }
\end{array}\right.
$$

which is then sent to succeeding decision element $\hat{x}_{i+1}$. The complexity of the decoding algorithm is determined essentially by the complexity of calculating LRs, which is $\mathrm{N}\left(1+\log _{2} N\right)=12$ for computing one round. For the initial

LRs, we calculate

$$
L\left(y_{i}\right)=\frac{W\left(y_{i} \mid 0\right)}{W\left(y_{i} \mid 1\right)} .
$$

For the down-polarizing system, the low level of LRs are obtained with a simple calculation using the reclusive formulas in (39), which gives 


$$
\begin{aligned}
& L_{2}^{(0)}\left(y_{k}\right)=L\left(y_{2 k-2}\right) \\
& L_{2}^{(1)}\left(y_{k}, \hat{x}_{2 k-2}\right)=\left(L\left(y_{2 k-1}\right)\right)^{1-2 \hat{x}_{2 k-2},}
\end{aligned}
$$

$\forall k \in\{1,2\}$. After that the high level of LRs can be calculated using the reclusive formulas in (41). A straightforward calculation yields

$$
\begin{aligned}
& L_{4}^{(0)}(y)=L_{2}^{(0)}\left(y_{1}\right), \\
& L_{4}^{(1)}\left(y, \hat{x}_{0}\right)=\left(L_{2}^{(0)}\left(y_{2}\right)\right)^{1-2_{\hat{x} 0},} \\
& L_{4}^{(2)}\left(y, \hat{x}_{I}\right)=\left(L_{2}^{(1)}\left(y_{1}, \hat{x}_{0}\right),\right. \\
& L_{4}^{(3)}\left(y, \hat{x}_{I}, \hat{x}_{2}\right)=\left(L_{2}^{(1)}\left(y_{2} \cdot \hat{x}_{0}+\hat{x}_{1}\right)\right)^{1-2_{\hat{x} 2}} .
\end{aligned}
$$

The LRs $L_{4}^{(i)}$ in (62) can be derived from (41), which can be found in Appendix C.

However, for the up-polarizing system the low level of LRs are given in [1] by

$$
\begin{aligned}
& L_{2}^{\prime(0)}(y k)=\frac{L\left(y_{2 k-2}\right) L\left(y_{2 k-1}\right)+1}{L(y 2 k-2)+L(y 2 k-1)} \\
& L_{2}^{\prime(1)}\left(y_{k}, \hat{x}_{2 k-2}\right)=\left(L\left(y_{2 k-2}\right)\right)^{1-2 \hat{x}_{2 k-2}} L\left(y_{2 k-1}\right),
\end{aligned}
$$

and the high level of LRs can be calculated as

$$
\begin{aligned}
& L_{4}^{\prime(0)}(y)=\frac{L_{2}^{\prime(0)}\left(y_{1}\right) L_{2}^{\prime(0)}\left(y_{2}\right)+1}{L_{2}^{\prime(0)}\left(y_{1}\right)+L_{2}^{(0)}\left(y_{2}\right)}, \\
& L_{4}^{(1)}\left(y, \hat{x}_{0}\right)=\left(L_{2}^{(0)}\left(y_{1}\right)\right)^{1-2 \hat{x} 0} L_{2}^{(0)}\left(y_{2}\right), \\
& L_{4}^{(2)}\left(y, \hat{\mathrm{x}}_{F}\right)=\frac{\left.L_{2}^{(1)}\left(y_{1}, \hat{x}_{0}\right) L_{2}^{\prime(1)}\left(y_{2}, \hat{x}_{0}+\hat{x}_{1}\right)\right)+1}{\left.L_{2}^{(1)}\left(y_{1}, \hat{x}_{0}\right)+L_{2}^{\prime(1)}\left(y_{2}, \hat{x}_{0}+\hat{x}_{1}\right)\right)} \\
& \left.L_{4}^{(3)}\left(y, \hat{\mathrm{x}}_{F}, \hat{x}_{2}\right)=L_{2}^{(1)}\left(y_{1}, \hat{x}_{0}\right)\right)^{1-2_{\hat{x} 2}} L_{2}^{(1)}\left(y_{2}, \hat{x}_{0}+\hat{x}_{1}\right) .
\end{aligned}
$$

So far we have calculated LRs of the polar system. The advantage of this OFDM depolarizing algorithm is due to the relations of two level LRs in coordination with the formulas in $(61,62,63,64)$. For example, two LRs $L_{4}^{(0)}\left(y_{t}\right)$ and $L_{4}^{(1)}\left(y_{t}, \hat{x}_{0}\right)$ are assembled from the same pair of $\operatorname{LRs} L_{2}^{(0)}\left(y_{1}\right)$ and $L_{2}^{(0)}\left(y_{2}\right)$, while the other two LRs $L_{4}^{(2)}\left(y_{t}, \hat{x}_{1}\right)$ and $L_{4}^{(3)}\left(y_{t}, \hat{\mathrm{x}}_{1} \hat{x}_{2}\right)$ are from $L_{2}^{(1)}\left(y_{1}, \hat{x}_{0}\right)$ and $L_{2}^{(1)}\left(y_{2}, \hat{\mathrm{x}}_{0}+\hat{x}_{1}\right)$ due to the symmetry properties of the FSF channels. In addition, two LRs $L_{2}^{(0)}\left(y_{k}\right)$ and $L_{2}^{(0)}\left(y_{k}, \hat{x}_{2 k-2}\right)$ are assembled from the LRs $\mathrm{L}^{(0)}\left(\mathrm{y}_{2 \mathrm{k}-2}\right)$ and $\mathrm{L}^{(0)}\left(\mathrm{y}_{2 \mathrm{k}-1}\right), \forall k \in\{1,2\}$. This process proposes an elegant approach for an accurate count of the total number of LRs that are required for a full description of the OFDM depolarizing algorithm, shown in Fig.4.

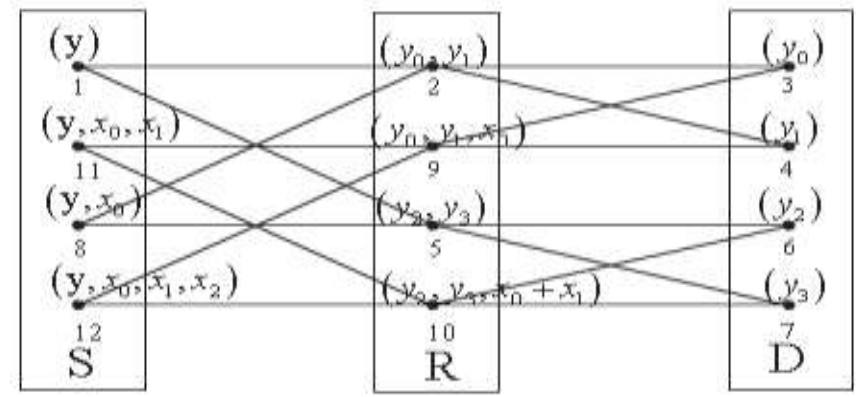

Fig. 3. Implementation of the successive cancellation decoder

Next we design an implementation of the SIC decoder for switching polar system. There are 12 nodes corresponding to LRs for decision elements $\hat{x}$. The depolarizing process carries out two crucial actions in the polar system, i.e., activating and responding [1]

- Step 1: It begins with the activating phase in the downpolarizing system, in which the first decision element $\hat{\mathrm{x}}_{1}$ from the leftmost column activates node 1 for the calculation of $L_{4}^{\prime(0)}(y)$ to decode $x_{1}$ of column vector $\mathrm{x}$, which in turn activates node 2 and node 5 to achieve a pair of LRs, $L_{2}^{(0)}\left(y_{1}\right)$ and $L_{2}^{(0)}\left(y_{2}\right)$. After that node 2 activates node 3 and node 4 , and node 5 activates node 6 and node7, respectively, for calculating two initiallevel pairs of $\mathrm{LRs}, \mathrm{L}^{(0)}\left(\mathrm{y}_{2 \mathrm{k}-2}\right)$ and $\mathrm{L}^{(0)}\left(\mathrm{y}_{2 \mathrm{k}-1}\right), \forall k \in\{1,2\}$. 
- Step 2: In the responding phase, node3 and node4 compute $\mathrm{L}^{(0)}\left(\mathrm{y}_{0}\right)$ and $\mathrm{L}^{(0)}\left(\mathrm{y}_{1}\right)$ using $(60)$, respectively, and pass them to their left-side two neighbors, node 2 and node 9 . Similarly, node 6 and node 7 , compute and pass the pair of LRs $\mathrm{L}^{(0)}\left(\mathrm{y}_{2}\right)$ and $\mathrm{L}^{(0)}\left(\mathrm{y}_{3}\right)$ to their left-side two neighbors, node 5 and node 10 , respectively.

- Step 3: In what follows, node2 and node5 compute $L_{2}^{(0)}\left(y_{1}\right)$ and $L_{2}^{(0)}\left(y_{2}\right)$ using (61) and pass the resulting pairs of LRs to its left-side two neighbors node1, respectively. After that, node1 compiles its response $L_{4}^{(0)}(y)$ to calculate $\hat{\mathrm{x}}_{0}$ according to (62). Consequently, node 1 sends $\hat{\mathrm{x}}_{0}$ to its neighbor node8, which is needed for calculating $\hat{\mathrm{x}}_{1}$. The yielded decision elements $\hat{\mathrm{x}}_{0}$ and $\hat{\mathrm{x}}_{1}$ are both passed to node 8 and node 12 that may generate $\hat{\mathrm{x}}_{2}$ and $\hat{\mathrm{x}}_{3}$. Fortunately, since $\hat{\mathrm{x}}_{2}$ and $x_{3}$ are the frozen bits that have low-reliability in the down-polarizing system, it is not necessary to generate $\hat{\mathrm{x}}_{2}$ and $\hat{\mathrm{x}}_{3}$ while directly setting $\hat{\mathrm{x}}_{2}=\hat{\mathrm{x}}_{3}=0$.

- Step 4: The depolarizing process switches to the up-polarizing system while calculating $\hat{\mathrm{x}}_{2}$ and $\hat{\mathrm{x}}_{3}$ with high reliability. In this phase $\hat{x}_{0}$ and $\hat{x}_{1}$ are the frozen bits that are directly set $\hat{x}_{0}=\hat{x}_{1}=0$. Consequently, $\hat{x}_{2}$ only activates node1 1 for computing $L_{4}^{\prime(2)}\left(y, \hat{\mathrm{x}}_{1}\right)$ based on (64), where $L_{2}^{\prime(1)}\left(y_{2}, \hat{\mathrm{x}}_{0}+\hat{x}_{1}\right) L_{2}^{\prime(1)}\left(y, \hat{x}_{1}\right)$ can be obtained from the response of node 9 and node 10 using (63). The decision element $\hat{\mathrm{x}}_{2}$ is then sent to node12 for $\hat{\mathrm{x}}_{3}$. The last decision element $\hat{\mathrm{x}}_{3}$ activates node12 for $x 3$ based on $L_{4}^{\prime(3)}\left(y, \hat{\mathrm{x}}_{1}, \hat{x}_{2}\right)$ in (64) without activating node 9 and node 10 . The algorithm continues in this manner until the receiver receives and decides the transmitted vector $\hat{\mathrm{x}}$.

We note that in the down-polarizing system it is not necessary to generate $\hat{\mathrm{x}}_{2}$ and $\hat{\mathrm{x}}_{3}$ since they are frozen bits that have low-reliability while transmitting in the down-polarizing channels. Similarly, in the up-polarizing system it is not necessary to generate $\hat{\mathrm{x}}_{0}$ and $\hat{\mathrm{x}}_{1}$ since they are frozen bits with the low-reliability while transmitting in the up-polarizing channels. In this way, the proposed depolarizing algorithm can be made while directly setting $\hat{\mathrm{x}}_{2}={ }^{\wedge} \mathrm{x} 3=0$ in the down-polarizing system and setting $\hat{\mathrm{x}}_{0}=\hat{\mathrm{x}}_{1}=0$ in the up-polarizing system. This decoding process continues until all information bits $\mathrm{x}$ are jointly decoded in the end. Next, we will show the BER performance behaviors of the polar system with simulation results. Thus, we can obtain $x$ from $\hat{x}$ in the polar system with the high-reliability.

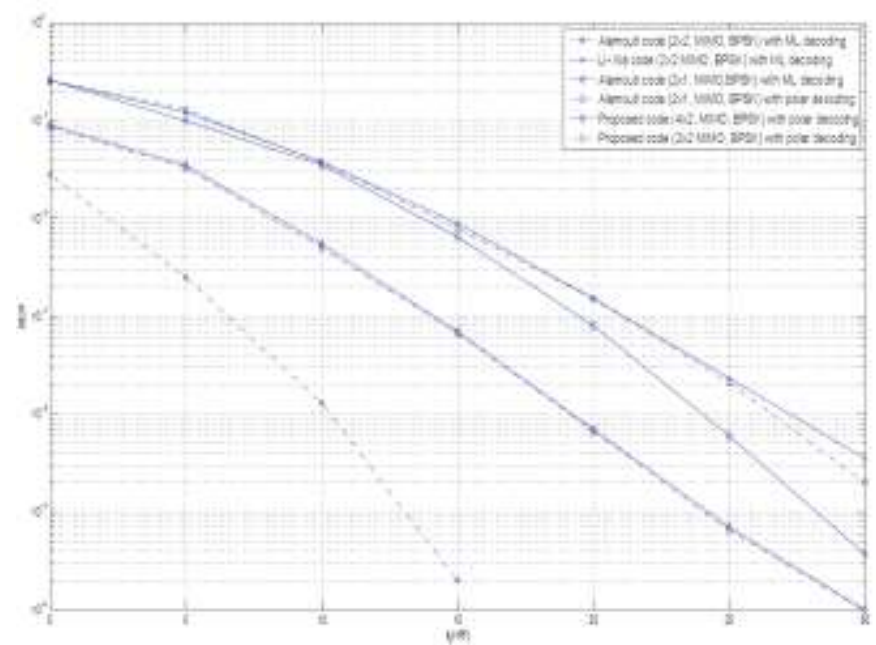

Fig. 4. BER performance behaviors with polar decoding. 


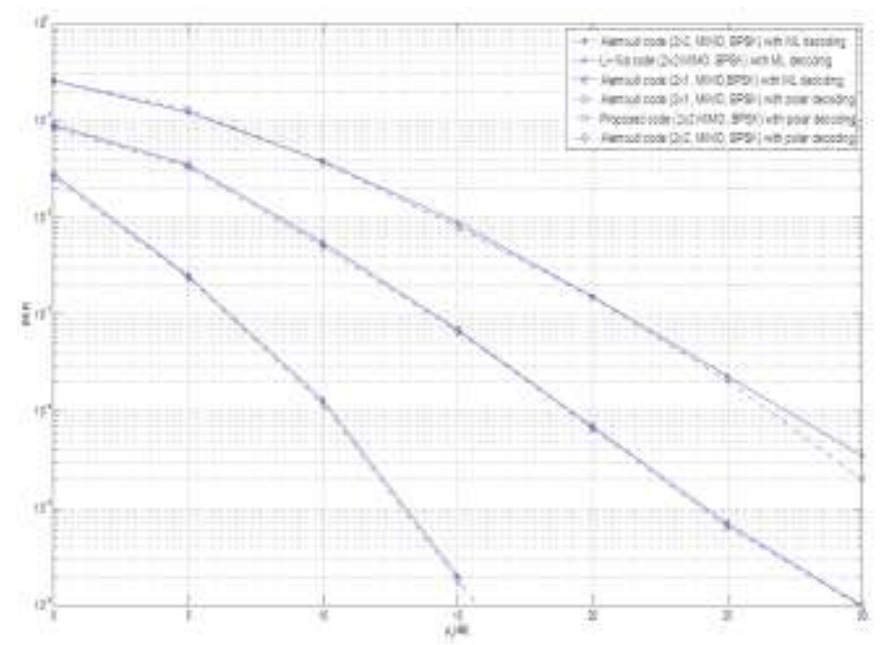

Fig. 5. BER performance comparison with ML decoding and polar decoding.

\section{E. Simulation Results}

According to the OFDM depolarizing algorithm with the SIC decoder for the polar system, we present some simulation results and compare their BER performance behaviors. We present the BER performance as functions of the transmit power $p_{t}$. We deploy the Alamouti code and the Jafarkhani code on each relay node $R_{i}$ while implementing the OFDM depolarizing techniques for the polarizing FSF channels. Therefore we can use the ML symbol-wise decoding, as well as the OFDM depolarizing algorithm in four time slots, where the data symbols in $A$ are drawn from BPSK constellation.

In Fig. 4, we present the BER curves of the stacked Alamouti code for four OFDM symbols transmitted at source node $S$. We consider the polar MIMO relay systems provided with transmission power $p_{t}$ for reference in terms of the power allocation strategy in (3). For the present polar system, it shows that the slope of the BER performance curve of the proposed PF scheme with the Alamouti code for the polar system via the OFDM depolarizing algorithm approaches the direct relay system without OFDM polarizing via the ML decoding when power $p_{t}$ increases. It implies that the PF scheme can achieve full diversity with the depolarizing algorithm. Furthermore, the BER performance behavior of the present polar system is a little better than that of the direct transmission approach which verifies our analysis of the transmission reliability of the polarized FSF channels. Simulations demonstrate that the proposed PF scheme with the OFDM depolarizing algorithm has a similar performance as that of the Alamouti scheme with the ML decoding for large transmission power $p_{t}$ when the depolarizing is applied at the receiver. In Fig. 6 it implies that the PF scheme can achieve full diversity in terms of the ML decoding and the OFDM depolarizing decoding. In this case, the BER performance behavior is much similar as those of the ML decoding, as stated in [11]. Fortunately, for the polar system, the BER performance of the decoder provided with the depolarizing algorithm outperforms that of the direct approach using the ML decoding.

\section{CONCLUSION}

In this paper, we have presented a simple design of the PF scheme based on two polarizing systems over the FSF channels, i.e., the down-polarizing system and the up-polarizing system. There are two polar coding processes for each polarizing system, including source polarizing and relay polarizing OFDM sequences. The present polar system has a salient recursiveness feature and can be decoded with the SIC decoder, which renders the scheme analytically tractable and provides a low-complexity coding algorithm while multiple

OFDM symbols are equipped. We analyze the BER performance and diversity of such systems based on the STF codes with the fixed size using the polarizing FSF channels, which tend to polarize with respect to the increasing reliability under certain OFDM combining and splitting operations. Simulations demonstrate that the proposed polar system has the similar BER performance behaviors as that of the STF codes, the stacked Alamouti code and the Jafarkhani code, but outperforms these STF codes in terms of the BER performance for large transmission power when the OFDM depolarizing algorithm is applied at the receiver.

\section{A. Proof of Theorem 3.1}

\section{APPENDIX}

We consider $4 \mathrm{~N}$ signals for four OFDM symbols $\left(x_{0}, x_{1}, x_{2}, x_{3}\right)$ embedded in (47) for the transmission in the down-polarizing system for the first two time slots. For each subcarrier $\epsilon \in \mathbb{Z}_{\mathrm{N}}$, we have $c_{0 \epsilon}=\left(x_{0 \epsilon}, x_{1 \epsilon}, x_{2 \epsilon}, x_{3 \epsilon}\right)^{T}$ and $c_{1 \epsilon}=\left(-x_{1 \epsilon}^{*}, x_{0 \epsilon}^{*},-x_{3 \epsilon}^{*}, x_{2 \epsilon}^{*}\right)^{T}$. According to the tree process of the Bhattacharyya para- 
meters for the recursive polarizing OFDMs in Fig.2(a), we achieve the respective information bits $\left(x_{0 \in}, x_{1 \in}\right)$ and $\left(-x_{1 \in}^{*}, x_{0 \in}^{*}\right)$ in two time slots after the CP removal and the $N$-point FFT operations at destination node $D$. The frozen bits are given by $\left(x_{2 \epsilon}, x_{3 \epsilon}\right)$ and $\left(-x_{3 \epsilon}^{*}, x_{2 \epsilon}^{*}\right)$ respectively. We do not need to decode frozen matrix $\mathrm{X}_{F}$ since it has been transmitted on channels with low reliability in the down-polarizing system. Therefore, after depolarizing the system we obtain the $4 \times 2$ originally transmitted matrix given by

$\hat{X}_{I}^{\prime}=\left(\begin{array}{ll}x_{2 €} & -x_{3 \epsilon}^{*} \\ x_{3 €} & x_{2 \epsilon}^{*}\end{array}\right)$.

Combining (65) and (66) in four time slots, we have $\hat{X}=\operatorname{diag}\left(\hat{X}_{A}, \hat{X}_{A}^{\prime}\right)$ from which the embedded signal matrix can be rewritten as

$\hat{\Lambda}(\mathrm{x})=\left(\begin{array}{ll}A\left(x_{0 \in}, x_{1 \in}\right. & 0_{2 \times 2} \\ 0_{2 \times 2} & A\left(x_{2 \epsilon}, x_{3 \in}\right)\end{array}\right)$,

where can be further decoded with the orthogonal STBC decoding algorithms. This completes the proof of Theorem 3.1.

\section{B. Proof of Theorem 3.2}

For each subcarrier of four OFDM symbols $\left(x_{0}, x_{1}, x_{2}, x_{3}\right)$ embedded in (52) for the transmission in both down-polarizing system. We transmit $\mathrm{X}$ in (53) in the first and third time slots for the polarizing MIMO relay system in (29). Namely, for each subcarrier $\varepsilon \in \mathbb{Z}_{\mathrm{N}}$, we have $c_{0 \epsilon}=\left(x_{0 \epsilon}, x_{1 \epsilon}, x_{2 \epsilon}, x_{3 \epsilon}\right)^{T}$ and $c_{3 \epsilon}=\left(-x_{2 \epsilon}^{+}, x_{3 \epsilon}^{+},-x_{0 \epsilon}, x_{1 \epsilon}\right)^{T}$ According to the tree process of the Bhattacharyya parameters for the recursive polarizing OFDMs in Fig.2(a), we achieve the respective information bits $\left(x_{0 €}, x_{1 \in}\right)$ and $\left(-x_{3 \in}^{+}, x_{2 \epsilon}^{+}\right)$in the first and third time slots after the $\mathrm{CP}$ removal and the $N$-point FFT operations at destination node $D$. The frozen bits are given by $\left(x_{2 \epsilon}, x_{3 \epsilon}\right)$ and $\left(x_{0 €}, x_{1 \epsilon}\right)$, respectively. After depolarizing the system we obtain the $2 \times 2$ originally transmitted matrix with high reliability given by

$\hat{X}_{I}^{\prime}=\left(\begin{array}{ll}-x_{3 €}^{+} & -x_{1 \in}^{+} \\ x_{2 €}^{+} & x_{0 €}^{+}\end{array}\right)$.

Combining (68) and (69) in four time slots, we have the embedded information matrix can be rewritten as After being depolarized and decoded at destination node $D$, we have

$\hat{J}(\mathrm{x})=\left(\begin{array}{cccl}x_{0 €} & 0 & -x_{2 \epsilon}^{+} & 0 \\ x_{1 \epsilon} & 0 & -x_{3 \epsilon}^{+} & 0 \\ 0 & -x_{3 \epsilon}^{+} & 0 & -x_{1 \epsilon}^{+} \\ 0 & x_{2 €}^{+} & 0 & x_{0 €}^{+}\end{array}\right)$.

We note that the depolarized information matrix $\hat{J}$ in (70) is a quasi-orthogonal matrices, which is equivalent to the Jafarkhani code if and only if $c_{0 \in} c_{1 \in}^{+} \neq c_{2 \epsilon} c_{3 \in}^{+}$. In fact, it is easy to prove that

$\hat{\mathrm{J}}(\mathrm{x}) \hat{\mathrm{J}}^{\mathrm{H}}(\mathrm{x})=$
$\left(\begin{array}{lccc}\left|c_{0 \epsilon}\right|^{2}+\left|c_{2 \epsilon}\right|^{2} & c_{0 \epsilon} c_{1 \epsilon}^{+}+c_{2 \epsilon} c_{3 \epsilon}^{+} & 0 & \\ c_{1 \epsilon} c_{0 €}^{+}+c_{3 \epsilon} c_{2 \epsilon}^{+} & \left|c_{1 \epsilon}\right|^{2}+\left|c_{3 \epsilon}\right|^{2} & 0 & \\ 0 & 0 & \left|c_{1 \epsilon}\right|^{2}+\left|c_{3 \epsilon}\right|^{2} & -c_{2 \epsilon} c_{3 \epsilon}^{+}+c_{0 €} c_{1 \epsilon}^{+} \\ 0 & 0 & -c_{3 \epsilon} c_{2 \epsilon}^{+}-c_{1 \epsilon} c_{0 €}^{+} & \left|c_{0 \epsilon}\right|^{2}+\left|c_{2 \epsilon}\right|^{2}\end{array}\right)$ From the above analysis, it implies that the

depolarized codes can be further decoded with the ML decoder or MMSE/ZF decoder for the quasi-orthogonal STBC [8]. However, whenever there is the constraint $c_{0} c_{1}^{+}=c_{2} c_{3}^{+}$for $\hat{J}(\mathrm{x})$, the yielded codes have an orthogonal structure and hence have the similar performance behaviors as that of the Alamouti code [7]. This completes the proof of Theorem 3.2.

\section{Proof of (62) from (41)}

In order to derive the LRs for the down-polarizing system, we deploy the calculation approach for the up-polarizing system suggested by Arikan [1]. 
For the simplicity of this proof, we consider the proof of the validity of the $\operatorname{LRs} L_{4}^{(i)}\left(y_{t}\right), \forall i \in \mathbb{Z}_{4}$, for decision element $\hat{x}_{i}$ in the depolarizing algorithm to illustrate the relationship of two level of LRs $L_{4}^{(i)}\left(y_{t}\right)$ in (62) and $L_{2}^{(i)}\left(y_{t}\right)$ in (61). According to the defined LR in (58), we use the recursive of the splitting transition probabilities $W_{4}^{(i)} \forall i \in \mathbb{Z}_{4}$, and thus achieve

$$
\begin{aligned}
& L_{4}^{(0)}(y)=\frac{W_{4}^{(0)}(y \mid 0)}{W_{4}^{(0)}\left(\left.y\right|_{1}\right)} \\
&= \frac{W_{2}^{(0)}\left(y_{1} \mid 0\right) W_{2}^{(0)}\left(y_{2} \mid 0\right)+W_{2}^{(0)}\left(y_{1} \mid 0\right) W_{2}^{(0)}\left(y_{2} \mid 1\right)}{W_{2}^{(0)}\left(y_{1} \mid 1\right) W_{2}^{(0)}\left(y_{2} \mid 1\right)+W_{2}^{(0)}\left(y_{1} \mid 1\right) W_{2}^{(0)}\left(y_{2} \mid 0\right)} \\
&= \frac{L_{2}^{(0)}\left(y_{1}\right) L_{2}^{(0)}\left(y_{2}\right)+L_{2}^{(0)}\left(y_{1}\right)}{1+L_{2}^{(0)}\left(y_{2}\right)} \\
&= L_{2}^{(0)}\left(y_{1}\right) \\
& L_{4}^{(1)}\left(y, \hat{\mathrm{x}}_{0}\right)=\frac{W_{4}^{(1)}\left(y, \hat{x}_{0} \mid 0\right)}{W_{4}^{(1)}\left(y, \hat{x}_{0} \mid 1\right)} \\
&= \frac{W_{2}^{(0)}\left(y_{1} \mid \hat{x}_{0}\right) W_{2}^{(0)}\left(y_{2} \mid \hat{x}_{0}+0\right)}{W_{2}^{(0)}\left(y_{1} \mid \hat{x}_{0}\right) W_{2}^{(0)}\left(y_{2} \mid \hat{x}_{0}+1\right)} \\
&=\left(L_{2}^{(0)}\left(y_{2}\right)\right)^{1-2 \hat{x}_{0}} \\
& L_{4}^{(2)}\left(y, \hat{\mathrm{x}}_{1}\right)=\frac{W_{4}^{(2)}\left(y, \hat{x}_{1} \mid 0\right)}{W_{4}^{(2)}\left(y, \hat{x}_{1} \mid 1\right)} \\
&= \frac{\sum_{x_{3}} W_{2}^{(1)}\left(y_{1}\left|x_{0}\right| 0\right) W_{2}^{(1)}\left(y_{2}, x_{0}+x_{1} \mid 0+x_{3}\right)}{\sum_{x_{3}} W_{2}^{(1)}\left(y_{1}\left|x_{0}\right| 1\right) W_{2}^{(1)}\left(y_{2}, x_{0}+x_{1} \mid 1+x_{3}\right)} \\
&= L_{2}^{(1)}\left(y_{1}, \hat{x}_{0}\right) \\
&= \frac{W_{2}^{(1)}\left(y_{1}, \hat{x}_{0} \mid \hat{x}_{2}\right) W_{2}^{(1)}\left(y_{2}, \hat{x}_{0}+\hat{x}_{1} \mid \hat{x}_{2}+0\right)}{W_{2}^{(1)}\left(y_{1}, \hat{x}_{0} \mid \hat{x}_{2}\right) W_{2}^{(1)}\left(y_{2}, \hat{x}_{0}+\hat{x}_{1} \mid \hat{x}_{2}+1\right)} \\
&\left.L_{4}^{(3)}\left(y_{2}, \hat{x}_{0}+\hat{x}_{1}\right)\right)^{1-2 \hat{x}_{2}} \\
&\left.W_{1}, \hat{\mathrm{x}}_{1} \hat{x}_{2}\right)=\frac{W_{4}^{(1)}\left(y, \hat{\mathrm{x}}_{1} \hat{x}_{2} \mid 0\right)}{W^{(1)}\left(y, \hat{\mathrm{x}}_{1} \hat{x}_{2} \mid 1\right)} \\
&=17
\end{aligned}
$$

In a similar way, we derive the formulas in (61) while calculating the low level LRs $L_{2}^{(i)}, \forall i \in \mathbb{Z}_{2}$, from (39). Namely, we obtain the relationship of two level LRs $L_{2}^{(i)}$ and LRs $L^{(i)}$ given by

$$
\begin{aligned}
& L_{2}^{(0)}\left(y_{k}\right)=\frac{W_{2}^{(0)}\left(y_{k} \mid 0\right)}{W_{2}^{(0)}\left(y_{k} l_{1}\right)} \\
& =\frac{W\left(y_{2 k-2} \mid 0\right) W\left(y_{2 k-1} \mid 0\right)+W\left(y_{2 k-2} \mid 0\right) W\left(y_{2 k-1} \mid 1\right)}{W\left(y_{2 k-2} \mid 1\right) W\left(y_{2 k-1} \mid 1\right)+W\left(y_{2 k-2} \mid 1\right) W\left(y_{2 k-1} \mid 0\right)} \\
& =\frac{L\left(y_{2 k-2}\right) L\left(y_{2 k-1}\right)+L\left(y_{2 k-2}\right)}{1+L\left(y_{2 k-1}\right)} \\
& =L\left(y_{2 k-2}\right)
\end{aligned}
$$




$$
\begin{aligned}
& L_{2}^{(1)}\left(y_{k}, \hat{x}_{2 k-2}\right)=\frac{W_{2}^{(1)}\left(y_{k}, \hat{x}_{2 k-2} \mid 0\right)}{W_{2}^{(1)}\left(y_{k}, \hat{x}_{2 k-2} \mid 1\right)} \\
& =\frac{W\left(y_{2 k-2} \mid \hat{x}_{2 k-2}\right) W\left(y_{2 k-1} \mid \hat{x}_{2 k-2}+0\right)}{W\left(y_{2 k-2} \mid \hat{x}_{2 k-2}\right) W\left(y_{2 k-1} \mid \hat{x}_{2 k-2}+1\right)} \\
& =\left(L\left(y_{2 k-1}\right)\right)^{1-2 \hat{x}_{2 k-2}}
\end{aligned}
$$

This completes the proof of our statement of derivation of LRs in (62) and (61).

\section{Acknowledgements}

This work was supported by the World Class University R32-2008-000-20014-0 NRF, Korea, and Fundamental Research 2010-0020942 NRF, Korea.

\section{References}

[1] Arikan, "Channel Polarization: A Method for Constructing Capacity-Achieving codes for Symmetric Binary-Input Memoryless Channel”, IEEE Trans. Inf. Theory, vol .55, pp.4366-4385, July 2009.

[2] Arikan, "Performance comparison of polar-codes and Reed-Muller codes". IEEE. Comm. Letters, vol.12, pp.447-449, June 2008.

[3] S. B. Korada E. S, as, o־glu and R. Urbanke, Polar Codes: "Characterization of Exponent, Bounds, and Constructions", IEEE Trans. Inf. Theory, vol.56, pp.6253-6264, Dec. 2010.

[4] M. H. Lee and E. Arikan, "Polar code and Jacket matrix", Seminar at Bilkent University, Turkey, August 2009.

[5] R. Mori and T. Tanaka, "Performance and Construction of Polar-codes on Symmetric Binary-Input Memoryless Channels", IEEE ISIT, Korea, June 2009.

[6] Y. Guo and M. H. Lee, "A Novel Channel Polarization on Binary Discrete Memoryless Channels”, IEEE ICCS, Singapore, Nov. 2010 .

[7] S. M. Alamouti, "A simple transmit diversity technique for wireless communications", IEEE J. Sel. Areas Commun., vol.16, pp.1451-1458, Oct. 1998.

[8] H. Jafarkhani, “A quasi-orthogonal space-time block codes”, IEEE Trans. Commun., vol.49, pp.1-4, Jan. 2001.

[9] W. Su and X.-G. Xia, and K. J. R. Liu, “A systematic design of high-rate complex orthogonal space-time block codes”, IEEE Trans. Inf. Theory, vol.8, pp.4340-4347, Jun. 2004.

[10] Y. Shang and X.-G. Xia, "Space-time block codes achieving full diversity with linear receivers", IEEE Trans. Inf. Theory, vol.54, pp.4528-4547, Oct. 2008.

[11] [11] X. Guo and X.-G. Xia, "On Full Diversity Space-Time Block Codes With Partial Interference Cancellation Group Decoding", IEEE Trans. Inf. Theory, vol.55, pp.4366-4385, Oct. 2009.

[12] Z. Li, X.-G. Xia and M. H. Lee, "A Simple Orthogonal Space-Time Coding Scheme for Asynchronous Cooperative Systems for Frequency Selective Fading Channels”, IEEE Trans. Commun, vol.58, pp.2219-2224 Aug. 2010.

[13] Tarokh, N. Seshadri, and A. R. Calderbank, Space-time codes for high data rate wireless communication: Performance criterion and code construction, IEEE Trans. Inf. Theory, vol. 44, no. 3, pp. 744-765, Mar. 1998.

[14] W. Zhang, X.-G. Xia, and K. B. Letaief, Space-time/frequency coding for MIMO-OFDM in next generation broadband wireless systems, IEEE Wireless Commun. Mag., vol. 14, no. 6, pp. 32-43, Jun. 2007.

[15] Y. Gong and K. B. Letaief, An efficient space-frequency coded OFDM system for broadband wireless communications, IEEE Trans. Commun., vol. 51, no. 12, pp. 2019-2029, Dec. 2003.

[16] W. Su, Z. Safar, M. Olfat, and K. J. R. Liu, Abtaining full-diversity space-frequency codes from space-time codes via mapping, IEEE Trans. Signal Process., vol. 51, no. 11, pp. 2905-2916, Nov. 2003.

[17] W. Su, Z. Safar, and K. J. R. Liu, Full-rate full-diversity space-frequency codes with optimum coding advantage, IEEE Trans. Inf. Theory, vol. 51, no. 1, pp. 229-249, Jan. 2005.

[18] W. Zhang, X.-G. Xia, and P. C. Ching, High-rate full-diversity space-time-frequency codes for broadband MIMO block-fading channels, IEEE Trans. Commun., vol. 55, no. 1, pp. 25-34, Jan. 2007.

[19] Y. Yan and Moon Ho Lee, "A Turbo-Like Symbol by Symbol Decoder for Randomly Distributed Space-Time Coding in Amplifyand-Forward Relay Networks", IEEE Comm. Letters, vol.14, Jan 2010.

[20] Z. Li, X.-G. Xia and B. Li, "Achieving full diversity and fast ML decoding via simple analog network coding for asynchronous twoway relay networks", IEEE Trans. Commun., vol.57, pp.3672-3681, Dec. 2009.

[21] S. Muhaidat, J.K. Cavers, and P. Ho, "Transparent Amplify-and-Forward Relaying in MIMO Relay Channels", IEEE Trans. Wireless Commun., vol.9, pp.3144-3154, Oct. 2010.

[22] Y. Jing and H. Jafarkhani, "Single and multiple relay selection schemes and their achievable diversity orders", IEEE Trans. Wireless Commun., vol.8, pp.1414-1423, Mar. 2009.

[23] Wang, J. Zhang and A. Host-Madsen, "On the capacity of MIMO relay channels", IEEE Trans. Inf. Theory, vol.51, pp.29-43, Jan. 2005 .

[24] Y. Rong, X. Tang and Y. Hua, “A Unified Framework for Optimizing Linear Nonregenerative Multicarrier MIMO Relay Communication Systems", IEEE Trans. Signal Processing, vol.57, pp.4837-4851, Dec. 2009.

[25] E. Shannon, “A mathematical theory of communication”, Bell Syst. Tech. J., vol. 27, pp. 379-423, 623-656, Jul.-Oct. 1948.

[26] Y. Jing and B. Hassibi, "Distributed Space-Time Coding in Wireless Relay Networks", IEEE Trans. Wireless Commun., vol.5, pp.3524-3536, Dec. 2006 